\title{
Survey of cold ionospheric outflows in the magnetotail
}

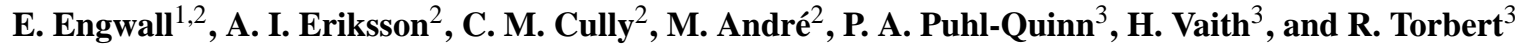 \\ ${ }^{1}$ Department of Physics and Astronomy, Uppsala University, Uppsala, Sweden \\ ${ }^{2}$ Swedish Institute of Space Physics, Uppsala, Sweden \\ ${ }^{3}$ Space Science Center, University of New Hampshire, Durham, NH, USA
}

Received: 24 February 2009 - Revised: 8 June 2009 - Accepted: 20 July 2009 - Published: 14 August 2009

\begin{abstract}
Low-energy ions escape from the ionosphere and constitute a large part of the magnetospheric content, especially in the geomagnetic tail lobes. However, they are normally invisible to spacecraft measurements, since the potential of a sunlit spacecraft in a tenuous plasma in many cases exceeds the energy-per-charge of the ions, and little is therefore known about their outflow properties far from the Earth. Here we present an extensive statistical study of cold ion outflows $(0-60 \mathrm{eV})$ in the geomagnetic tail at geocentric distances from 5 to $19 R_{E}$ using the Cluster spacecraft during the period from 2001 to 2005 . Our results were obtained by a new method, relying on the detection of a wake behind the spacecraft. We show that the cold ions dominate in both flux and density in large regions of the magnetosphere. Most of the cold ions are found to escape from the Earth, which improves previous estimates of the global outflow. The local outflow in the magnetotail corresponds to a global outflow of the order of $10^{26}$ ions $\mathrm{s}^{-1}$. The size of the outflow depends on different solar and magnetic activity levels.
\end{abstract}

Keywords. Magnetospheric physics (Magnetosphereionosphere interactions; Magnetotail; Polar cap phenomena)

\section{Introduction}

Low-energy outflows from the high-latitude ionosphere consist of ion bulk outflows, such as the polar wind and auroral bulk outflows. The outflows in the cusp region form the cleft ion fountain (Lockwood et al., 1985), which is transported over the polar cap by anti-sunward convection and mixes with the other low-energy outflows. When travelling farther out along the magnetic field lines into the lobes, it will be difficult to distinguish the different sources of the outflows, and

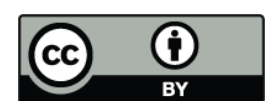

Correspondence to: E. Engwall (erik.engwall@irfu.se) we will here refer to them only as high-latitude ion outflows. In addition to the low-energy outflows, there exist energetic outflows, such as ion beams and conics. The general characteristics of the bulk and energetic outflows were summarised in Yau and André (1997).

Supersonic low-energy outflows from the polar ionosphere were first predicted by Axford (1968) and Banks and Holzer (1968), and were named the polar wind because of similarities to the solar wind. The first direct measurements of the polar wind were achieved in the late 1960s by Explorer 31, which found $\mathrm{H}^{+}$outflows at 500 and $3000 \mathrm{~km}$ with velocities up to $15 \mathrm{~km} / \mathrm{s}$ (Hoffman, 1970). Since the first measurements of the polar wind, a number of different spacecraft have probed the high-latitude ion outflows close to their outflow region in the ionosphere. ISIS 2 confirmed the outflow of $\mathrm{H}^{+}$, but also found evidence for outflows of $\mathrm{He}^{+}$and $\mathrm{O}^{+}$ (Hoffman et al., 1974; Hoffman and Dodson, 1980). With DE-1 the first extensive studies of the polar wind were made (Nagai et al., 1984; Chandler et al., 1991). The current understanding of the high-latitude ion outflows, including the polar wind, can mainly be attributed to a wealth of studies from Akebono (Abe et al., 1993, 1996, 2004; Cully et al., 2003a) and Polar (e.g. Moore et al., 1997; Su et al., 1998; Chappell et al., 2000; Lennartsson et al., 2004; Liemohn et al., 2005; Huddleston et al., 2005; Peterson et al., 2006). More details on the previous measurements of the high-latitude ion outflows can be found in the recent review articles by Yau et al. (2007) and Moore and Horwitz (2007).

Using Polar and Akebono measurements as initial conditions in global outflow simulations, the high-latitude ionospheric outflows have been predicted to continue far out through the magnetotail lobes and feed the plasma sheet or escape beyond (Chappell et al., 2000; Huddleston et al., 2005; Moore et al., 2005; Cully et al., 2003b). The plasma sheet feeding by the ionospheric outflows is especially evident during active times with southward IMF, which leads to anti-sunward convection above the polar cap. During

Published by Copernicus Publications on behalf of the European Geosciences Union. 
northward IMF, the convection stagnates, and the intensity of the ion outflows decreases. At these times the plasma sheet is essentially fed by the low-latitude boundary layer (Moore et al., 1999a).

Even though simulations have suggested the continuation of the high-latitude outflows far out in the magnetotail, detections of low-energy ions in this region have been rare. The main reason is that the potential of a sunlit spacecraft in this low-density region will reach several tens of volts positive, and thus prevent low-energy ions from reaching the spacecraft. Most of the few measurements far away from the Earth have been conducted by the Geotail spacecraft at hundreds of $R_{E}$ downtail. Mukai et al. (1994), Hirahara et al. (1996) and Seki et al. (1998) found cold ion streams with high enough energy to overcome the spacecraft potential. In 2001 the Polar orbit had precessed to the equatorial plane, making it possible to measure outflowing ions with energy below $300 \mathrm{eV}$ as far as $9.5 R_{E}$ down the tail (Liemohn et al., 2005). However, the ions with the lowest energies were still hidden because of the high positive spacecraft potential, typically above $20 \mathrm{~V}$ in the tail lobes.

The ions with the very lowest energies have seldom been detected in the magnetotail. With Geotail in eclipse, rendering a negative spacecraft potential, Seki et al. (2003) were able to detect cold ions coexisting with the hot plasma sheet population. Olsen (1982) made similar measurements of cold ions when two different spacecraft (Applied Technology Satellite 6 and SCATHA) were in eclipse. By artificially regulating the potential of Polar, Su et al. (1998) were able to make a statistical study of the low-energy ion outflow at $8 R_{E}$ above the northern pole.

Engwall et al. (2006a) reported the first measurement of low-energy ion flows with energy of the order of $10 \mathrm{eV}$ at a geocentric distance as far as $18 R_{E}$ using two different methods on the Cluster spacecraft: (1) using a conventional ion detector in a special low-energy mode, and under simultaneous operation of artificial spacecraft potential control, and (2) using a new method, which detects the ions through electric field measurements of the large wake created behind the spacecraft in this flowing plasma. In a recent study, Engwall et al. (2009) used the latter method for a statistical study in the lobes during 2002. This study reached much higher altitudes and covered a much larger volume in space than ever before. It was shown that the high-latitude ion outflows remain cold far out in the lobes and dominate in this region in both flux and density. The total outflow from the Earth was found to be of the order of $10^{26}$ ions/s.

In this paper, we extend the work in Engwall et al. (2009) to include substantially more data covering several years during the declining phase of the solar cycle: 2001-2005. We also investigate the distribution in space of cold flowing ions and the dependence of their outflow properties on different controlling parameters, such as geomagnetic and solar activity. The paper is structured as follows: Sect. 2 explains the method of detection, its limitations and the basis for error analysis of the method. The next section describes the selection criteria for the data used, and Sect. 4 gives the general properties and the distribution in space of the outflow. In Sect. 5 we examine the dependence of the outflow on solar and magnetic activity. Further, in Sect. 6 we discuss possible error sources and our results in the context of previous measurements at lower altitudes.

\section{Method}

\subsection{Method description}

The new method is based on measurements from two different electric field instruments on-board the Cluster satellites to detect the enhanced ion wake arising behind a positively charged spacecraft in a cold tenuous plasma. The direction of the wake gives the flow direction of the ion outflow and indirectly the magnitude of the flow velocity.

An enhanced wake will form if the bulk ion flow energy $m v_{\mathrm{i}}^{2} / 2$ not only exceeds the thermal energy $K T_{\mathrm{i}}$, but also is lower than the equivalent energy of the spacecraft potential $e V_{\mathrm{sc}}$, i.e.

$K T_{\mathrm{i}}<\frac{m v_{\mathrm{i}}^{2}}{2}<e V_{\mathrm{sc}}$.

The ion wake will be filled with electrons, whose thermal energy, in contrast to that of the ions, is higher than the ram kinetic energy. When the wake is enhanced by the spacecraft potential, the wake becomes sufficiently large for the negative charge density to create an appreciable negative potential, and thus also a substantial local wake field close to the spacecraft. The electric field instrument EFW (Gustafsson et al., 1997) with probes mounted on wire booms separated by $88 \mathrm{~m}$ is able to detect this wake field (Eriksson et al., 2006; Engwall et al., 2006b). Figure 1 shows a schematic picture of the wake formation. Its physical properties as well as its effect on a double probe instrument have been verified by particle-in-cell simulations (Engwall et al., 2006b). The other electric field instrument on Cluster, EDI (Paschmann et al., 1997), is based on a completely different technique: the electric field is inferred by measuring the drift of artificially emitted high-energy $(\mathrm{keV})$ electrons as they gyrate back to the spacecraft under the influence of the ambient magnetic field. In the lobes the gyroradius of the EDI electrons is several $\mathrm{km}$ while the wake length scale is on the order of $100 \mathrm{~m}$, and EDI will be left essentially unaffected by the local wake field (Eriksson et al., 2006).

The wake electric field is thus given by $\boldsymbol{E}^{\mathrm{w}}=\boldsymbol{E}^{\mathrm{EFW}}-\boldsymbol{E}^{\mathrm{EDI}}$ and can be used to derive the flow velocity of the ions. These cold ions are normally invisible to conventional ion spectrometers on-board spacecraft, since the ion energy per charge seldom exceeds the spacecraft potential. Assuming 
(a) $m v_{\mathrm{i}}^{2} / 2>K T_{\mathrm{i}}, m v_{\mathrm{i}}^{2} / 2>>e V_{\mathrm{sc}}$
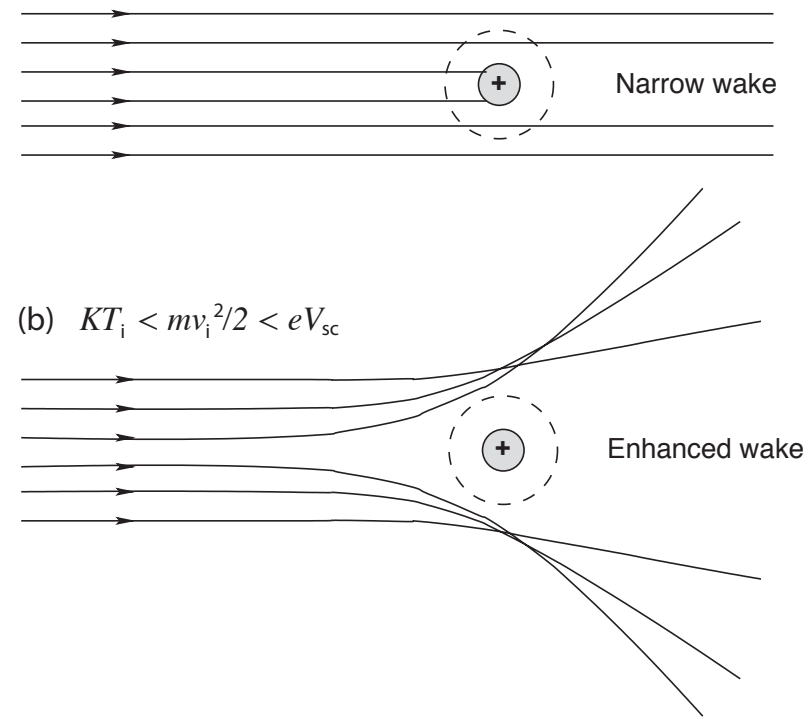

Fig. 1. Sketch of wake formation in supersonic ion flows $\left(K T_{\mathrm{i}}<m v_{\mathrm{i}}^{2} / 2 ; K T_{\mathrm{e}}>m v_{\mathrm{e}}^{2} / 2\right)$. (a) For ion flow energies much higher than the equivalent spacecraft potential, $V_{\mathrm{sc}}$, the wake has the typical transverse size of the spacecraft (narrow wake case). (b) For ion flow energy below $V_{\mathrm{sc}}$, ions will scatter off the positive electrostatic potential from the spacecraft, creating an enhanced wake. In both cases, the electrons will fill the wake, since their thermal energy is higher than the ram kinetic energy.

that the ions are unmagnetized on the wake length scale, the wake electric field is in the flow direction, i.e.

$\boldsymbol{E}^{\mathrm{W}}=g \boldsymbol{u}$

where $\mathbf{u}$ is the flow velocity and $g$ a scalar function dependent on e.g. spacecraft potential and ion temperature. Provided that EDI data is good and the frozen-in condition applies, the perpendicular velocity is given by $\boldsymbol{u}_{\perp}=\boldsymbol{E}^{\mathrm{EDI}} \times \boldsymbol{B} / \boldsymbol{B}^{2}$. The parallel velocity can then be obtained by decomposition of $\boldsymbol{E}^{\mathrm{W}}$ in the two measured components $x$ and $y$ in the spacecraft spin plane, and by subsequent division of $E_{x}^{\mathrm{w}}$ with $E_{y}^{\mathrm{w}}$ :

$\frac{E_{x}^{\mathrm{W}}}{E_{y}^{\mathrm{W}}}=\frac{g u_{\perp, x}+g u_{\|} B_{x} / B}{g u_{\perp, y}+g u_{\|} B_{y} / B}$.

The $\mathrm{x}$-axis points as close to sunward as possible, while the $\mathrm{y}$-axis is perpendicular to the $\mathrm{x}$-axis and is directed towards dusk. Rearranging Eq. (3) and writing in vector form gives

$\mathbf{u}_{\|}=\frac{E_{x}^{\mathrm{w}} u_{\perp, y}-E_{y}^{\mathrm{w}} u_{\perp, x}}{E_{y}^{\mathrm{w}} B_{x}-E_{x}^{\mathrm{w}} B_{y}} \mathbf{B}$.

Figure 2 describes the method schematically. The validity of the model was verified for one case in the magnetotail at $18 R_{E}$ (Engwall et al., 2006a), comparing the derived flow velocity with simultaneous measurements from the ion detector CIS (CODIF) on another of the Cluster satellites under

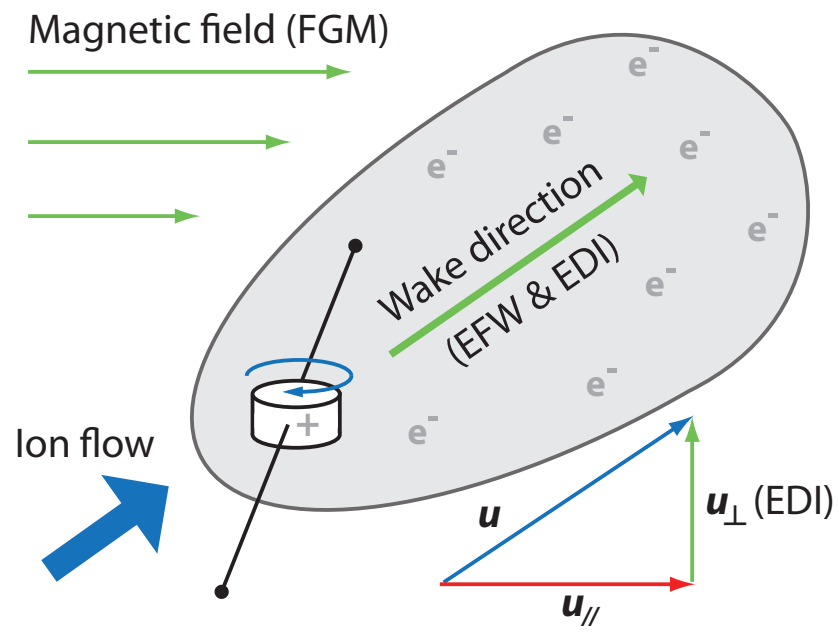

Fig. 2. Method to derive ion flow velocity on Cluster. The flow velocity can be derived from the negative wake created behind a positively charged spacecraft with knowledge of three quantities (shown in green): (1) the direction of the wake, (2) the ambient magnetic field, $\mathbf{B}$, and (3) the velocity perpendicular to $\mathbf{B}$. The direction of the wake, which is in the flow direction, is given by the wake electric field $\mathbf{E}^{\mathrm{W}}=\mathbf{E}^{\mathrm{EFW}}-\mathbf{E}^{\mathrm{EDI}}$. The EFW double probes separated by $88 \mathrm{~m}$ wire booms will be affected by the wake field, whereas the EDI electron beam will not. The magnetic field is obtained from the FGM instrument on-board Cluster and the perpendicular velocity is inferred from EDI: $\mathbf{u}_{\perp}=\mathbf{E}^{\mathrm{EDI}} \times \mathbf{B} / B^{2}$. Equation (4) is used to derive the parallel velocity, $\mathbf{u}_{\|}$, and the total ion flow velocity, $\mathbf{u}$, is thus also obtained. (The sketch shows the simplified case, when the magnetic field and the wake is in the spacecraft spin plane.)

special circumstances: low spacecraft potential due to operation of the artificial spacecraft control, ASPOC (Torkar et al., 2001), and operation of CODIF (Rème et al., 2001) in lowenergy mode $(0.7-25 \mathrm{eV})$. Events where these circumstances are fulfilled on one spacecraft at the same time as wakes are seen in the electric field signature on another spacecraft are rare. However, this event study includes enough data to establish the model as a good method to derive the ion flow velocity.

To obtain the ion flux, $n u$, we need a measure of the ion density, $n$. Since the ion population is invisible to the particle detectors, we use the spacecraft potential, $V_{\mathrm{sc}}$, to estimate the ion density. Pedersen et al. (2008) have recently derived a formula for low-density plasmas and it has been used for a statistical study of the total density distribution in the lobes (Svenes et al., 2008). We refine the total density relation of Pedersen et al. (2008) with a daily normalization to the solar ultraviolet (UV) flux to compensate for the effect of daily solar variations (Eriksson and Winkler, 2008), and apply it to the cold ion data set. (The solar UV flux is represented by the $\mathrm{F}_{10.7}$ index, which is the integrated emission from the solar disc at $10.7 \mathrm{~cm}$ wavelength $(2800 \mathrm{MHz})$ measured at local noon at Penticton, Canada.) For 2002 through 2005 we use the formula for 2004 in Pedersen et al. (2008) normalised to the mean value of $\mathrm{F}_{10.7}$ during 2004: 


$$
\begin{aligned}
n^{\mathrm{EFW}}(t)=\frac{\mathrm{F}_{10.7}(\mathrm{t})}{<\mathrm{F}_{10.7}>_{2004}} & {\left[3 \exp \left(-\frac{V_{\mathrm{sc}}}{8.8 \mathrm{~V}}\right)\right.} \\
+ & \left.0.05 \exp \left(-\frac{V_{\mathrm{sc}}}{30 \mathrm{~V}}\right)\right] \mathrm{cm}^{-3} .
\end{aligned}
$$

This can be justified by the fact that for high spacecraft potentials the relations from Pedersen et al. (2008) for the years 2002 through 2005 normalised to the respective yearly mean of $\mathrm{F}_{10.7}$ deviate very little from each other. However, for 2001, there is a substantial difference, which can be explained by the short period of operation up to that date. The surface coating of a spacecraft and its conducting properties will change during the first year of operation in space, giving slightly different spacecraft potential for a given plasma density than during later years. For 2001 we therefore adopt the formula for 2001 of Pedersen et al. (2008) normalised to the mean value of $\mathrm{F}_{10.7}$ during that year:

$$
\begin{aligned}
n_{2001}^{\mathrm{EFW}}(t)=\frac{\mathrm{F}_{10.7}(\mathrm{t})}{<\mathrm{F}_{10.7}>_{2001}} & {\left[10 \exp \left(-\frac{V_{\mathrm{sc}}}{7.4 \mathrm{~V}}\right)\right.} \\
+ & \left.0.05 \exp \left(-\frac{V_{\mathrm{sc}}}{30 \mathrm{~V}}\right)\right] \mathrm{cm}^{-3} .
\end{aligned}
$$

From Eqs. (4) through (6), the ion flux is determined, and the method can be used for a statistical study of ion flux far out in the geomagnetic tail lobes, where it has previously been invisible to spacecraft. The results are shown in Sect. 4.

To quantify the global outflow from the Earth we map the local flux values to a reference altitude of $1000 \mathrm{~km}$. (At this altitude, the magnetic field is around $37 \mu \mathrm{T}( \pm 1 \mu \mathrm{T})$ for all relevant magnetic latitudes.) The mean value of the mapped flux is then multiplied by the estimated area of the two polar caps. Assuming that the polar cap extends to $70^{\circ}$ geomagnetic latitude, the area at $1000 \mathrm{~km}$ is given by $2 \times 2 \pi\left(R_{E}+1000 \mathrm{~km}\right)^{2}\left(1-\cos \left(90^{\circ}-70^{\circ}\right)\right)=4.1 \times 10^{17} \mathrm{~cm}^{2}$. The global outflow can also be estimated from the mean of the local flux values multiplied by the area of the crosssection of the tail lobe, where Cluster detects cold ions (see Fig. 3). This area can be described as a circle with diameter $28 R_{E}$ minus a rectangle of $28 R_{E}$ width and $2 R_{E}$ height to account for the plasma sheet. Since the region where cold ions exist could extend beyond the Cluster orbit, this gives a good lower estimate of the total outflow. The two estimates give very similar results, and we therefore choose to use the first method in this paper.

\subsection{Method limitations}

Even if the method is very powerful, there are a few limitations: (1) EDI data must exist, which requires sufficiently strong magnetic fields, (2) measurement is not possible during ASPOC operation, since it removes the wake signature that the method relies on, (3) the wake must not be completely perpendicular to the spin plane, since we need to measure a component of the wake direction in the spin plane,
(4) there is no possibility to detect cold ions coexisting with hot plasma, since the hot ions will fill the wake and cancel the wake electric field, and (5) we have no provision to distinguish between different ion species.

If the "frozen-in" condition applies and centrifugal acceleration dominates (Cladis, 1986), point (5) poses no problem for the velocity calculation, since both the perpendicular and parallel velocities are independent of mass. However, it is possible that the ion species are subject to different acceleration processes resulting in mass-dependent velocities. The derived parallel velocity is then a weighted average of the parallel velocities of the different particles. However, our method is much more sensitive to lighter ion species, since their lower energy will make them more affected by the spacecraft potential and thus create a larger wake. Moreover, hydrogen is the dominant ion species in the low-energy high-latitude ion outflows ( $\mathrm{Su}$ et al., 1998). Our calculated velocity can thus be regarded as the proton velocity, and later comparison of the properties of the outflowing ions to previous measurements will therefore only regard the hydrogen component of the flow. However, the measured density is the total of all ion species, and when calculating the proton flux we lower the density by a factor of 0.8 , since protons on average constitute around $80 \%$ of the cold plasma density at high altitudes (Su et al., 1998). The factor of 0.8 gives only a rough estimate of the proton density, and it should be noted that the composition of the ionospheric outflow can vary substantially with geomagnetic and solar activity (e.g. Cully et al., 2003a). This means that the calculated flux can be subject to larger errors than the velocity (see Sect. 6.1).

In addition to these limitations, there is no possibility to distinguish between two counterstreaming ion populations, since we only see the resulting wake. To know what our method yields in a situation with counterstreaming ion beams, we would need to know the function $g(u)$ in Eq. (2) above. Let us assume that this is weakly dependent on $u$, and also that it is proportional to the ambient density $n$. The latter assumption is reasonable as the smallest dimension of the wake stays below the Debye length. In this case, Poisson's equation directly leads to a wake potential that depends linearly on the excess electron density, which is just $n$ because the wake potential (in volts) is much smaller than the electron temperature (in electronvolts), as can be seen in the simulations by Engwall et al. (2006b). It follows that the wake forming on each side of the spacecraft will give an apparent electric field $\boldsymbol{E}_{w}$ approximately proportional to the flux $n \boldsymbol{u}$ of the population causing it. As the wake potentials are considerably smaller than both the spacecraft potential and the ion bulk energy, the trajectories of counterstreaming ion beams are not much affected by the wakes generated by their opposite partner. Therefore the wakes superpose linearly, and under the assumptions above, this would be true also for the wake electric field signatures in our data. The form of the function $g(u)$ is not known, but as long as it does not depend strongly on $u$, we will still get an estimate of the 
net flux, particularly if the bulk speeds of the counterstreaming populations are similar. We therefore expect that our flux estimates are reasonable first approximations to the net flux, even in cases of counterstreaming ions, or indeed for any superposition of cold ion streams, whatever their direction.

\subsection{Error analysis}

To reduce errors in the data set, we remove potentially bad data. First, we adopt a threshold on $\mathrm{F}_{10.7}$, which disregards points below $100 \times 10^{-22} \mathrm{~W} \mathrm{~m}^{-2} \mathrm{~Hz}^{-1}$, since the low photoemission during such conditions sometimes causes the EFW probes (fed with a $140 \mathrm{nA}$ bias current) to saturate. Of the total data set $30 \%$ of the data points are removed, and of the data points from 2005 as many as $82 \%$ of the data points had to be removed. Each data point is the average value during one spacecraft spin with period of $4 \mathrm{~s}$.

Equation (4) is analysed for each point to get an estimate of the mean error in the velocity calculation. If the error exceeds $40 \%$ the data point is removed. For the EFW error estimates, we base these on findings from comparisons of EFW to EDI and CIS data and statistics obtained during the production of the EFW data set for the Cluster Active Archive (Khotyaintsev et al., 2009, and Yuri Khotyaintsev, personal communication, 2009). The accuracy of the $E_{y}$ component is very good, with a typical difference to EDI or CIS mean values of better than $0.1 \mathrm{mV} / \mathrm{m}$. The $E_{x}$ component is subject to a higher uncertainty, due to variation of the observed sunward offset caused by asymmetric photoemission (Pedersen et al., 1998). For Cluster 3, which we will use in the subsequent statistical study, an average value of the sunward offset is around $1.6-1.7 \mathrm{mV} / \mathrm{m}$ with a spread (double standard deviation) less than $0.5 \mathrm{mV} / \mathrm{m}$. The sunward offset has been compensated for before the analysis and the spread of $0.5 \mathrm{mV} / \mathrm{m}$ is used for the uncertainty in $E_{x}$. In the EDI data each data point contains not only information about the electric field and the perpendicular velocity, but also magnitude and angle errors on the drift step, which is used to calculate the electric field and perpendicular velocity. We transform these errors to errors on the electric field and perpendicular velocity for use in the analysis. For the total error on the wake field, $\Delta E^{\mathrm{w}}$, EFW contributes more (median values of $\Delta E_{x}^{\mathrm{EDI}}$ and $\Delta E_{y}^{\mathrm{EDI}}$ are slightly below 0.04 and $0.1 \mathrm{mV} / \mathrm{m}$, respectively). The errors in the magnetic field data from FGM is assumed to be $\Delta B_{x, y, z}=0.1 \mathrm{nT}$.

Some of the ions with lowest velocity will be missed by our method, since we need a drift energy in the spin plane larger than the ion temperature $(\sim 0.2-0.3 \mathrm{eV}$ in the polar wind (Yau et al., 2007)) to detect a wake. To reduce statistical errors in the velocity calculation, we have therefore applied a $10 \mathrm{~km} / \mathrm{s}(0.5 \mathrm{eV}$ for protons) limit on the bulk ion speed in the spin plane. Also, when the drift energy is well above the spacecraft potential, we will not detect any wake field. This puts an upper limit at around $60 \mathrm{eV}(110 \mathrm{~km} / \mathrm{s}$ for protons).
The effects of the error removal on the statistical study are discussed in Sect. 6.1.

\section{Data selection}

For a statistical study we use data from Cluster 3 from 3 July to 3 November during 2001-2005. This gives four months of data each year, centred on 3 September, when the spacecraft apogee of $19.6 R_{E}$ was in the geomagnetic tail exactly behind the Earth. All parts of orbits with geocentric distances beyond $5 R_{E}$ are chosen for the analysis. We consider cold ions to be detected when the wake field exceeds $2 \mathrm{mV} / \mathrm{m}$, which is well above the noise level.

The original data set consists of almost 1100000 data points. After removal of spins where $\mathrm{F}_{10.7}$ is too low, the number of spins is reduced to around 765000 . Out of these, over 540000 data points contain wakes. The total data set for velocity analysis consists after error removal of almost 180000 data points. Since each data point is the average during the $4 \mathrm{~s}$ spacecraft spin period, all 180000 data points which have been used for the statistics of the flowing ions correspond to almost $200 \mathrm{~h}$ of data. Figures 3-5 are based on detection of wakes in the 765000 data points, whereas Figs. 6-12 contain information on the velocity and thus based on the 180000 data points.

\section{Properties and distribution in space of ion outflow}

\subsection{Distribution in space}

Figure 3 displays the data we have analysed in the GSM $X-Z$, $X-Y$, and $Y-Z$ planes. The measurements cover a large volume in the geomagnetic tail lobes, where the ions previously have been invisible. In the upper panel, each dot corresponds to $30 \mathrm{~min}$ of data and the colour shows the occurrence percentage of cold flowing ions. Missing data points can mainly be attributed to lack of EDI data, which is especially frequent in the plasma sheet. In this region, EDI operation is limited by the weakness and variability of the magnetic field and by high ambient electron fluxes at the EDI beam energy of $1 \mathrm{keV}$. The lower panel shows the same data as in the upper panel but projected on the coordinate planes. The grid is divided in $2 R_{E}$ by $2 R_{E}$ bins. Each bin has to contain at least $1 \%$ of the mean number of points in a bin to obtain a colour.

As can be clearly seen, the cold flowing ions are in general very frequent: in total over $71 \%$ of the data points contain cold ions. However, on the flanks (large $\left|Y_{\mathrm{GSM}}\right|$ ), there are few wakes detected. This suggests that the cold flowing ions originate in the polar cap proper at high-latitudes. Magnetic field lines in the flanks map back to the auroral region, where ion outflows are common, but then at much higher energies than those we are able to observe.

In Fig. 4, the percentage of all events where cold ions have been detected are displayed as a function of $X_{\mathrm{GSE} / \mathrm{GSM}}$, 


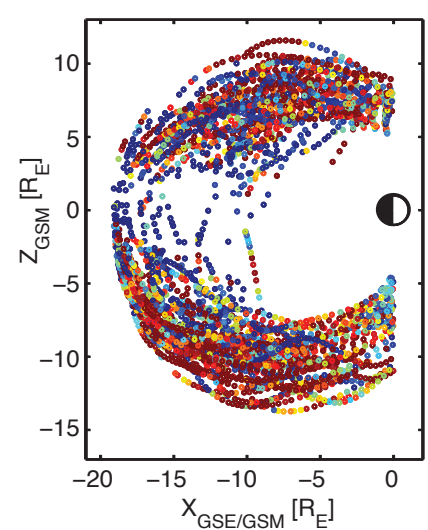

(a)

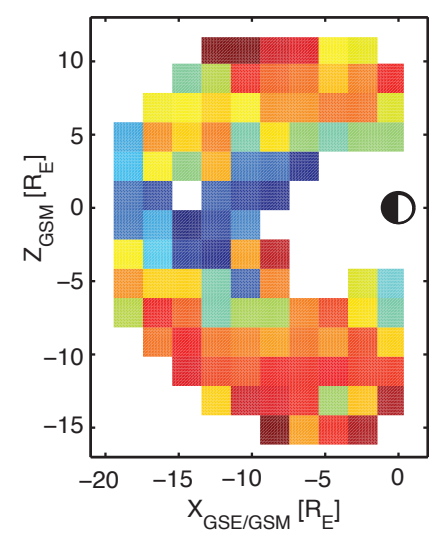

(d)

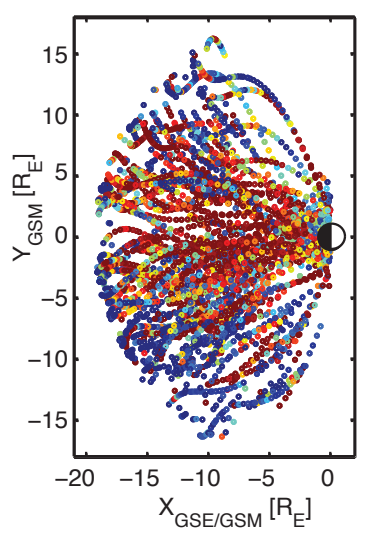

(b)

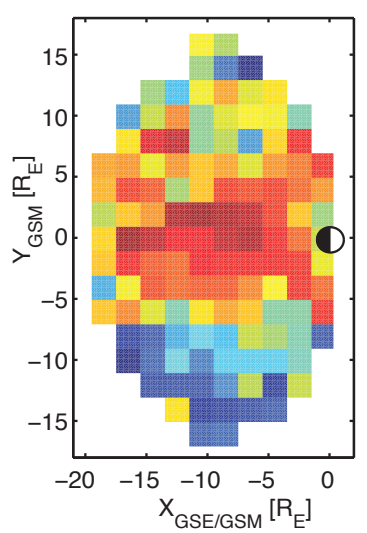

(e)

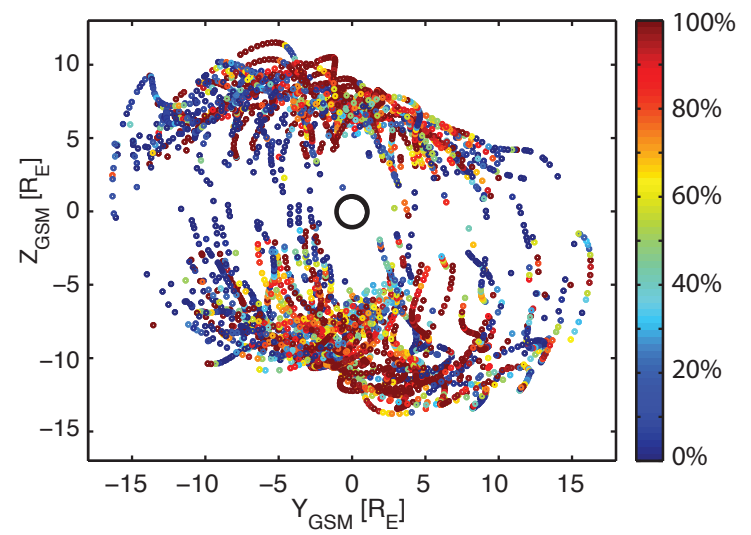

(c)

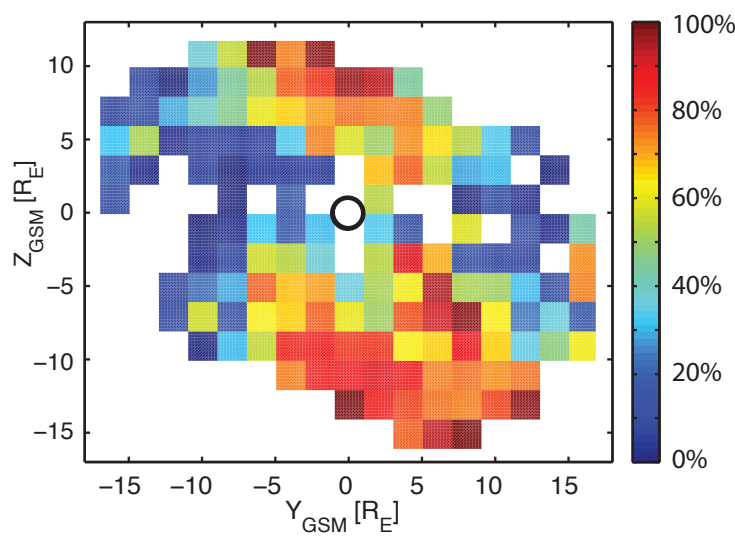

(f)

Fig. 3. Occurrence of cold ions. Detection of cold ions in the geomagnetic tail in the GSM $X-Z, X-Y$, and $Y-Z$ planes. In the upper panel, each point corresponds to $30 \mathrm{~min}$ of data. The colour shows the occurrence percentage of cold ions at the point. The lower panel shows the same data grouped in bins of $2 R_{E}$ by $2 R_{E}$. A bin is coloured if it contains at least $1 \%$ of the mean number of points per bin. (The total data set consists of 765000 points.)

$Y_{\mathrm{GSM}}$, and $Z_{\mathrm{GSM}}$. The number of data points in each bin is shown in red. As was identified in Fig. 3, the occurrence of cold ions is lower toward the geomagnetic tail flanks (middle panel). However, the variation with distance is even more obvious when sorting on $Z_{\mathrm{GSM}}$, with many more wakes detected for high $\left|Z_{\mathrm{GSM}}\right|$. We examine this relation more closely by plotting the wakes detected as a function of the distance along $Z_{\mathrm{GSM}}$ from the neutral sheet (see Fig. 5), which is estimated using the Tsyganenko 1995 model (Tsyganenko, 1995) (scaling parameters in $R_{E}$ set to: $R_{H}=8$, $\Delta X=4, G=10$, and $L_{w}=10$ ). The variation is smoother than in Fig. 4 and it is clear that around the neutral sheet the detection probability is very low.

Closer to the neutral sheet it is more probable that the ions already are energised in the outflow source region. This happens for example for ion outflows from the auroral region, which are directly connected to the plasma sheet. Also, the probability for the ions to get heated once they enter the plasma sheet is high. Thus, in the central plasma sheet, data points are not only fewer, but there are also very few wakes detected. In addition, even if the cold ions are exempted from heating, the hot plasma sheet population will prevent the creation of a wake inhibiting measurement of cold ions. Detection of cold ions coexisting with the hot plasma sheet population, as revealed by Olsen (1982) and Seki et al. (2003) is therefore not possible with our method.

We now examine the dependence of velocity, density and flux as a function of distance from the Earth (see Fig. 6). The velocity increases almost linearly with distance, which is expected due to centrifugal acceleration. Simulations of outflow under centrifugal acceleration have shown that the ions will attain comparable velocities as in our study when reaching high altitudes and continue to flow out with a moderate velocity increase with distance (Cladis, 1986). The density and flux, on the other hand, decrease approximately exponentially with distance. Examining the total outflow from the ionosphere in ions/s as a function of distance, we see that this quantity remains more or less constant, especially if we disregard the first and the last five data points. This can serve as a self-consistency check of the method, showing that mass is conserved along the outflow trajectory. 

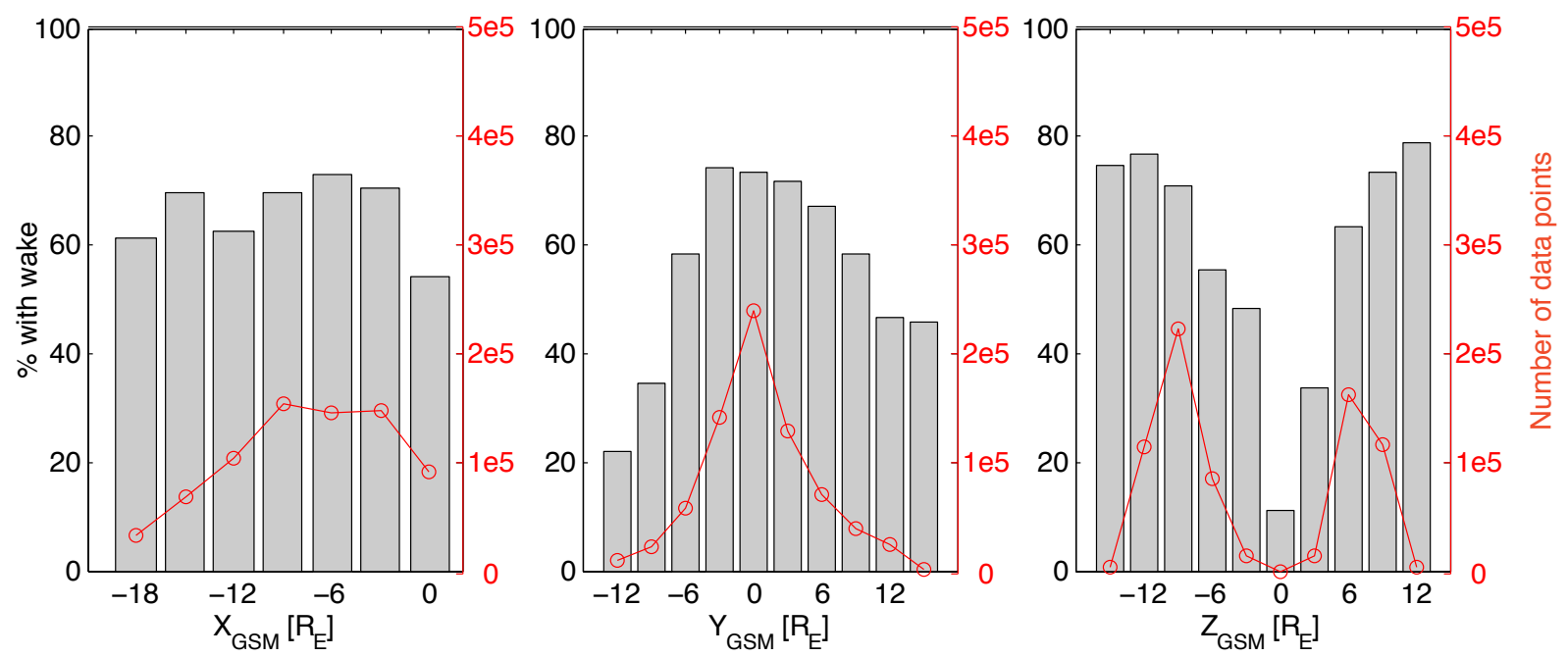

Fig. 4. Detection probability as a function of distance. Percentage of all events where cold ions have been detected as a function of $X_{\mathrm{GSE} / \mathrm{GSM}}, Y_{\mathrm{GSM}}$, and $Z_{\mathrm{GSM}}$. The red points show the total number of data points in the respective region. (The total data set consists of 765000 points.)

\subsection{Density and velocity map}

In Fig. 7, a spatial map of the density and flow velocity of the cold flowing ions is shown in the GSM $X-Z$ and $X-Y$ planes. The planes are divided in a mesh with grid size $2 R_{E}$ in each direction. For each plane we average over all data at particular values of $X$ and $Z$, and $X$ and $Y$, respectively. To get enough counting statistics, only bins containing over approximately 350 points ( $30 \%$ of the mean number of points in a bin) are taken into account. This high threshold is applied in order to suppress random effects from single orbits. The figure confirms the results from Fig. 6 with higher densities closer to the Earth and higher velocities farther away. The flow pattern is very much what would be expected of ionospheric outflow, and is in accordance with previous simulations (e.g. Delcourt et al., 1989, and Huddleston et al., 2005). However, the current study is the first to use measurements in situ in the lobes beyond $9 R_{E}$ to map densities and velocities of the high-latitude low-energy (order $10 \mathrm{eV}$ ) outflow.

In the GSM $X-Z$ plane there is an asymmetry in both velocity and density with higher values in the Northern Hemisphere. This could be explained by orbital asymmetry and seasonal variations; the measurements are centred on 3 September, which means that the data are shifted by around 20 days from autumnal equinox towards northern summer. During summer the amount of escaping photoelectrons from the upper atmosphere increases, resulting in a larger ambipolar electric field and thus also a larger ion outflow (Tam et al., 1995). Large seasonal variations of the electron density have been seen at high altitude above the polar cap with Polar, and the variations are particularly steep around the equinoxes (Laakso et al., 2002).

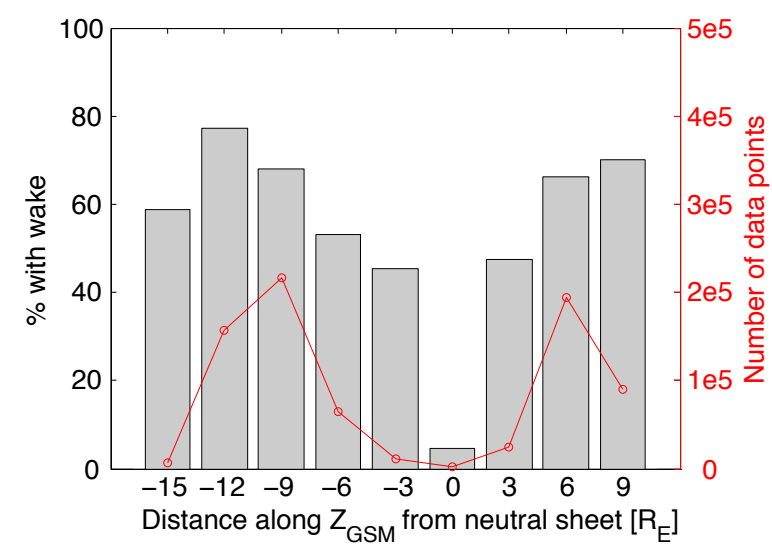

Fig. 5. Detection probability as a function of the distance from the neutral sheet. Percentage of all events where cold ions have been detected as a function of distance along $Z_{\mathrm{GSM}}$. The red points show the total number of data points in the respective region. (The total data set consists of 765000 points.)

In the GSM $X-Y$ plane, the line where the velocity points only in the $X_{\mathrm{GSM}}$-direction is shifted towards negative values of $Y_{\mathrm{GSM}}$. This is explained by the fact that the outflowing ions will $\mathbf{E} \times \mathbf{B}$-drift along equipotential contours connected to the cross-polar cap electric field. As has been clearly mapped out by Haaland et al. (2007), the symmetry line of the potential contours is rotated toward dusk on the night-side for positive (negative) IMF $B_{Y}$ on the Northern (Southern) Hemisphere. For negative (positive) IMF $B_{Y}$ on the Northern (Southern) Hemisphere, the convection is either symmetric along the noon-midnight line or becomes stagnated. The net drift will therefore be directed from dawn 


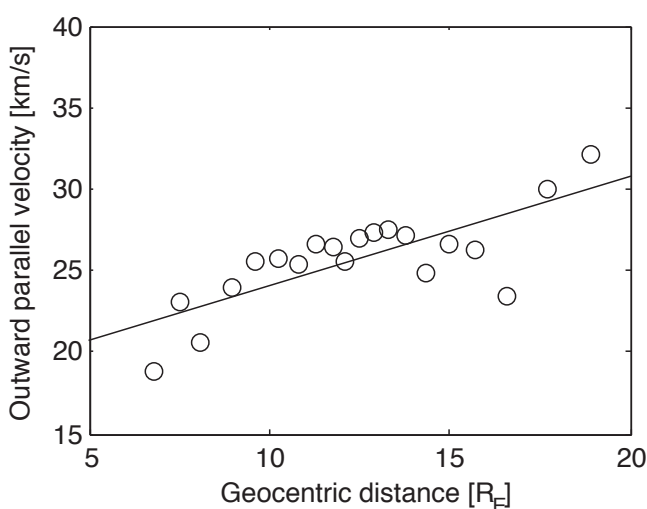

(a)

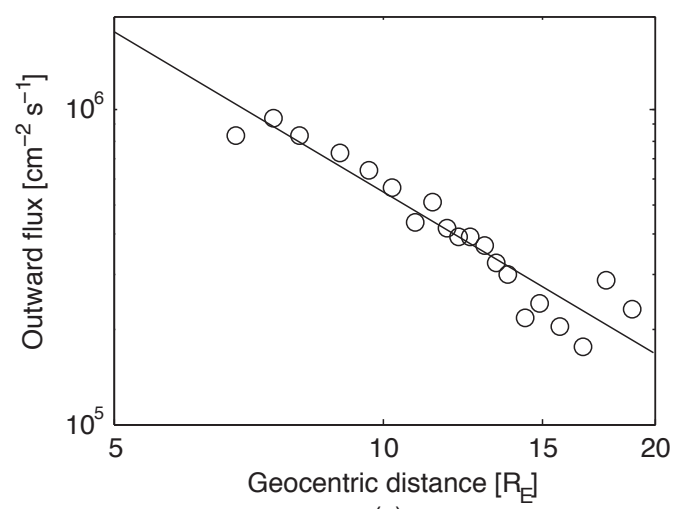

(c)

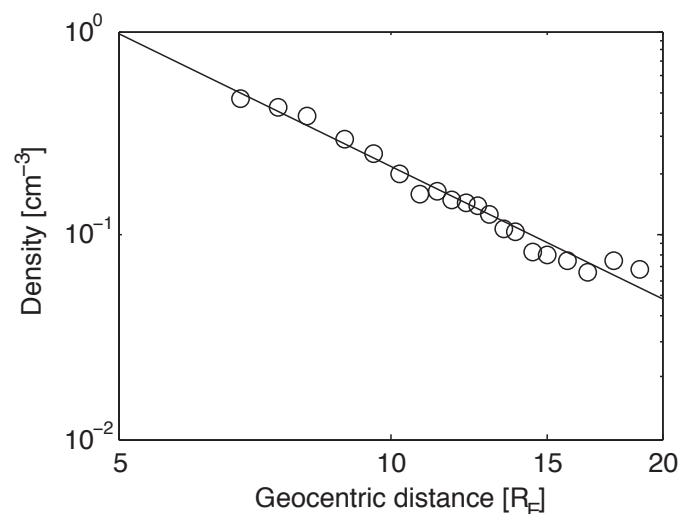

(b)

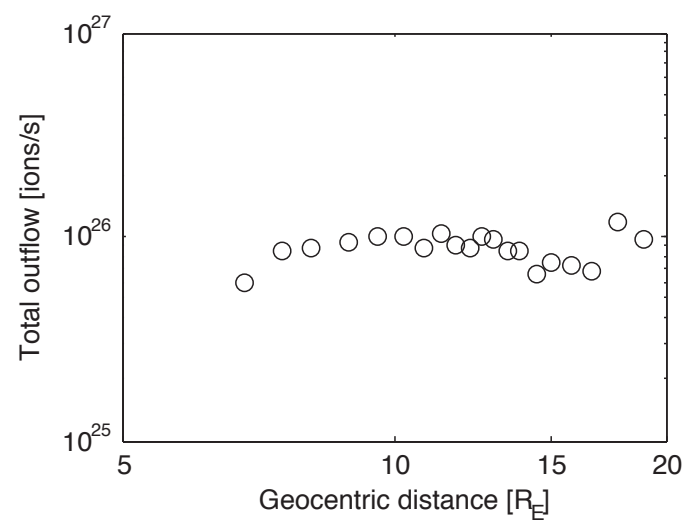

(d)

Fig. 6. Altitude dependence. Dependence on altitude of the outward parallel velocity (a), the ion density (b), the outward flux (c), and the global outflow (d). (The total data set consists of 180000 points.)

to dusk, which is what we see in the lower panel of Fig. 7 and also in Fig. 3e, where more cold ions are detected on the dusk side. The asymmetry in velocity will also result in a density asymmetry. Comparing to electron densities from Polar (Laakso et al., 2002), we see the same behaviour with higher densities for positive GSM $Y$.

\subsection{General properties}

The general properties of the cold outflowing ions are summarised in histograms in Fig. 8. As explained in Sect. 2.2, the measured velocity is the proton velocity and the derived properties displayed in the histograms pertain therefore to the protons. Figure 8 a shows the parallel velocity along the magnetic field and Fig. $8 \mathrm{~b}$ displays the magnetic-field-aligned velocity away from the Earth. Negative (positive) parallel velocities shown in yellow (blue) in Fig. 8a correspond to outflows in the Northern (Southern) Hemisphere, while negative outward parallel velocities (Fig. 8b) correspond to inward moving ions. Note that we miss some of the ions with lowest velocity, since we need a drift energy in the spin plane larger than the ion temperature to detect a wake, as discussed in Sect. 2.3. The second row of Fig. 8 shows the plasma density inferred from the spacecraft potential, the outward flux and the outward flux mapped back to the ionosphere. The measured density is the density of all ion species, so the outward proton flux is obtained from the outward parallel velocity times the density corrected by a factor of 0.8 , as explained previously. The ionospheric flux has been mapped to $1000 \mathrm{~km}$ and can be used to quantify the global outflow, as described in Sect. 2.1.

The mean (median) values of the outward velocity, density, and outward flux of $26 \mathrm{~km} / \mathrm{s}(24 \mathrm{~km} / \mathrm{s}), 0.18 \mathrm{~cm}^{-3}$ $\left(0.10 \mathrm{~cm}^{-3}\right)$, and $3.5 \times 10^{5} \mathrm{~cm}^{-2} \mathrm{~s}^{-1}\left(2.1 \times 10^{5} \mathrm{~cm}^{-2} \mathrm{~s}^{-1}\right)$ show that we measure a tenuous and moderately outflowing plasma. The mean (median) value of the mapped ionospheric flux is $1.8 \times 10^{8} \mathrm{~cm}^{-2} \mathrm{~s}^{-1}\left(1.2 \times 10^{8} \mathrm{~cm}^{-2} \mathrm{~s}^{-1}\right)$. This is consistent with the theoretical limiting flux value for protons on the order of $3 \times 10^{8} \mathrm{~cm}^{-2} \mathrm{~s}^{-1}$ at $1000 \mathrm{~km}$ at solar minimum. The flux is limited by the rate of production of protons in the topside ionosphere, as well as the rate of Coulomb collisions with other ions (Yau et al., 2007). The mapped ionospheric flux corresponds to a global outflow of $0.74 \times 10^{26} \mathrm{ions} / \mathrm{s}\left(0.50 \times 10^{26} \mathrm{ions} / \mathrm{s}\right)$.

The density is low, but it is still much higher than expected from particle detectors, which detect very few ions. 
This is also higher than measured in the study by Svenes et al. (2008), who obtained plasma densities in the range $0.007-0.091 \mathrm{~cm}^{-3}$ for two thirds of the measurement points. It is natural that our values are higher, since we identify events with outflowing ions and estimate the density for those events, while Svenes et al. (2008) estimate the mean of the density for all events during the observation time.

The low mean value of the outward parallel velocity shows that we indeed measure the continuation of the high-latitude ion outflows, since $26 \mathrm{~km} / \mathrm{s}$ corresponds to a kinetic energy of $3.5 \mathrm{eV}$ for protons. To be able to detect the ions, a wake must exist and the temperature of the cold ions must therefore be lower than the flow energy. Thus, the temperature cannot be higher than a few electronvolts, which excludes all other ion sources except the ionosphere. The low outflow energy also shows that the ions remain unenergised throughout the lobes, which shows that the ions have been only very weakly accelerated or heated along the outflow path.

The mean (median) value of the speed of $32 \mathrm{~km} / \mathrm{s}$ $(29 \mathrm{~km} / \mathrm{s})$ shows that the convection towards the plasma sheet is slower than the velocity along magnetic field lines. As can be seen in Fig. 8b, only a few events with inward flowing ions are detected. This means that there are almost no return fluxes of cold ions towards the Earth, which in turn implies that those ions that eventually reach the plasma sheet are efficiently heated or escape tailward.

\section{Variations with solar and magnetic activity}

\subsection{Source data}

We now sort our data by different solar wind parameters and geomagnetic indices. Variations due to these parameters mainly arise in the ionosphere, and we therefore use the mapped ionospheric flux for this analysis. First we examine the dependence on solar radiation, represented by the daily $\mathrm{F}_{10.7}$ values. For the variations with magnetic activity we use the 3-hourly index $K_{\mathrm{p}}$. The solar wind data is $1 \mathrm{~h}$ resolution OMNI2 data with ACE and WIND as source spacecraft. OMNI2 data is time shifted to the magnetopause, while our observations are made farther away from the magnetopause. However, we apply no further time shift, because of the difficulty to determine the time of flight from the ionosphere, where the solar wind variations mainly take effect, to the point of observation. The time of flight could be as long as a couple of hours, so the subsequent analysis will only be sensitive to variations on the time scale of one hour or more. The source data is interpolated to the time line of the Cluster data.

\subsection{Solar radiation dependence}

With data from 2001 through 2005, we have the possibility to study the solar radiation dependence during almost half a solar cycle; during this time the $F_{10.7}$ values range from

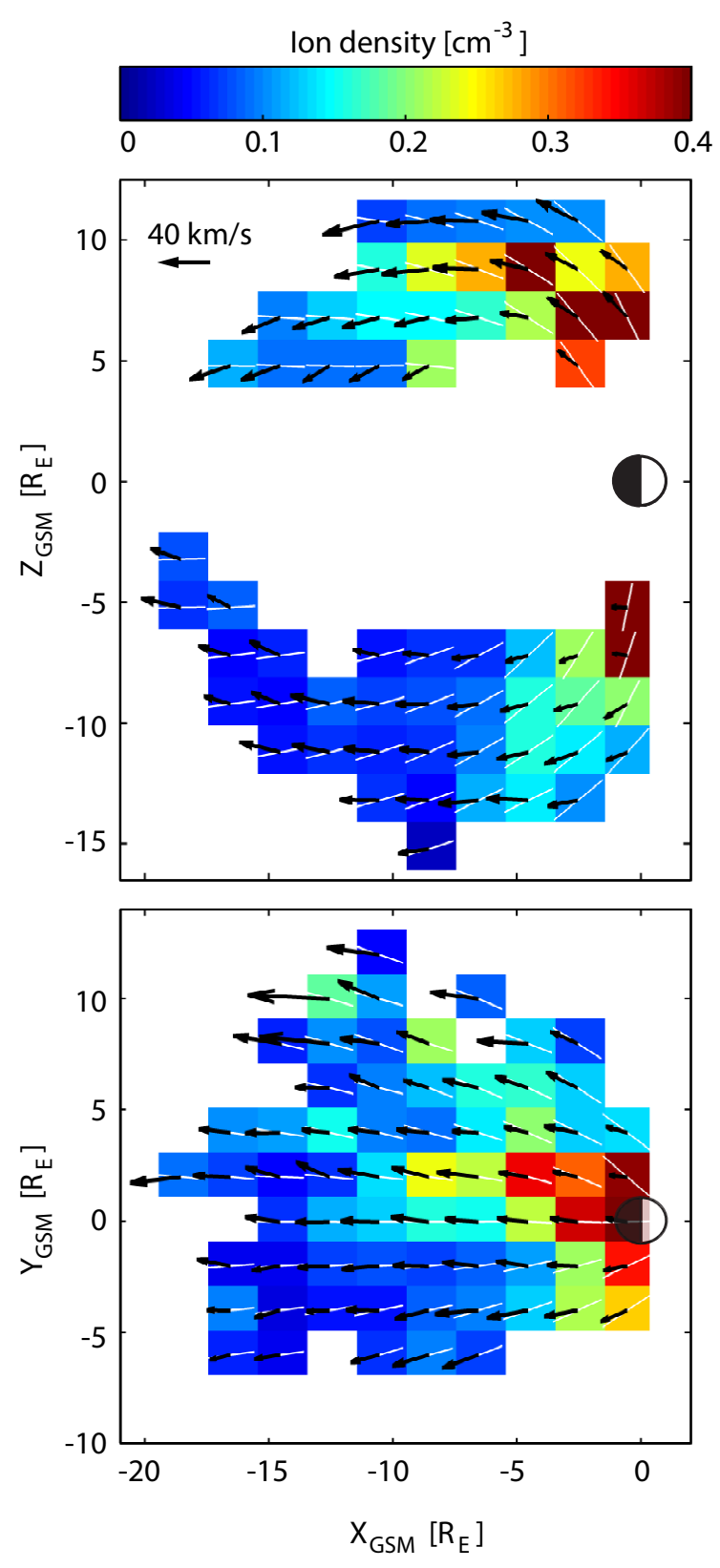

Fig. 7. Density and velocity maps. The mean density and velocity are shown in the GSM $X-Z$ and $X-Y$ planes. The data are grouped in $2 R_{E}$ by $2 R_{E}$ bins. A bin is coloured if the number of points in the bin exceeds approximately 350 (30\% of the mean number of points in a bin). The direction of the magnetic field is shown in white. (The total data set consists of 180000 points.)

71 to $285 \times 10^{-22} \mathrm{~W} \mathrm{~m}^{-2} \mathrm{~Hz}^{-1}$, with a mean value around $142 \times 10^{-22} \mathrm{~W} \mathrm{~m}^{-2} \mathrm{~Hz}^{-1}$. Unfortunately, as explained in Sect. 2.3, we have to remove data where $\mathrm{F}_{10.7}$ is below 100 . However, as can be seen in Fig. 9, a clear dependence for the remaining values can still be seen. The mapped flux increases by a factor of 3 from the lowest to the highest value. 


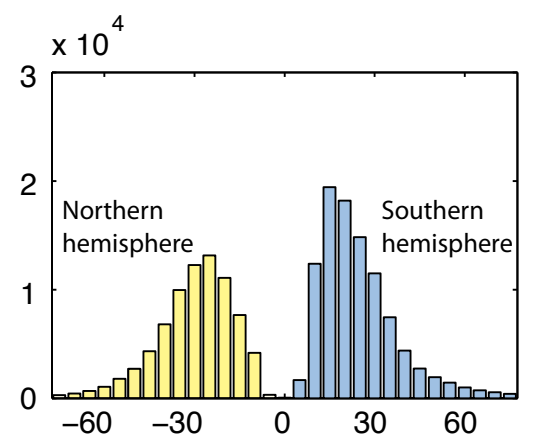

(a) Parallel velocity $[\mathrm{km} / \mathrm{s}]$

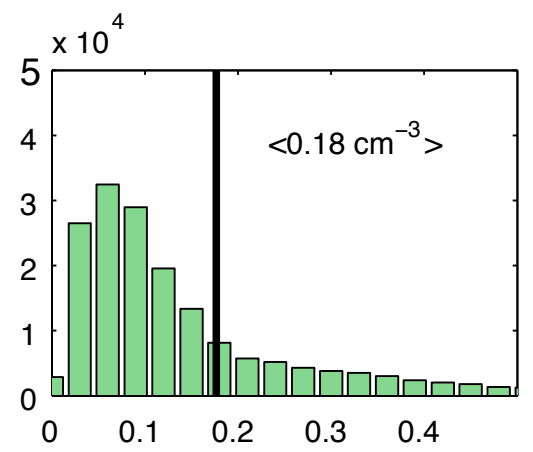

(d) Density $\left[\mathrm{cm}^{-3}\right]$

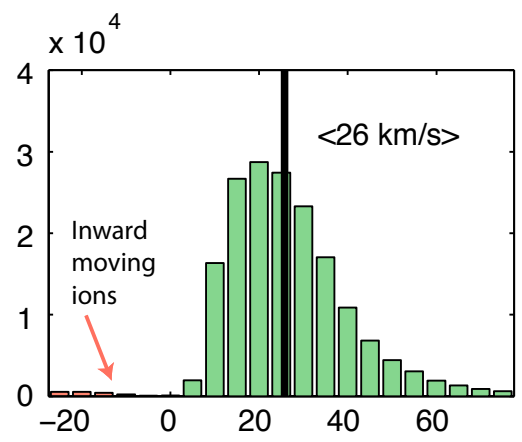

(b) Outward parallel velocity $[\mathrm{km} / \mathrm{s}]$

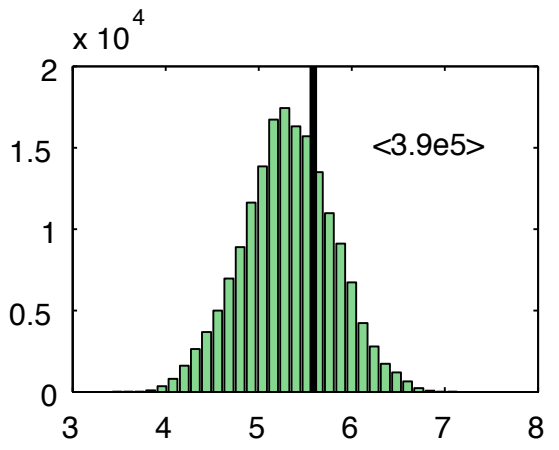

(e) Outward flux $\log \left[\mathrm{cm}^{-2} \mathrm{~s}^{-1}\right]$

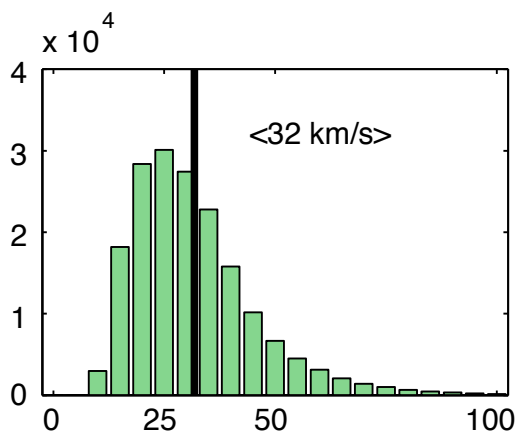

(c) Total flow speed [km/s]

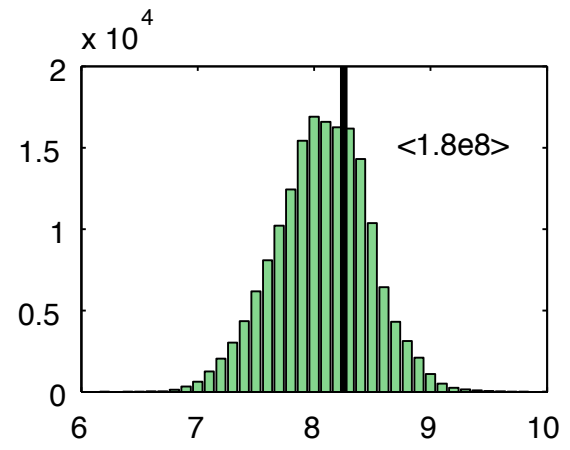

(f) Mapped flux $\log \left[\mathrm{cm}^{-2} \mathrm{~s}^{-1}\right]$

Fig. 8. Properties of the high-latitude ion outflow. (a) Velocity along the magnetic field. (b) The magnetic field aligned velocity away from the Earth. Negative outward parallel velocities correspond to inward moving ions, which are only seen in a few events. (c) Total speed of the ions. (d) Density derived from the spacecraft potential measured by EFW. (e) Outward proton flux obtained from the outward parallel velocity times the density corrected by a factor of 0.8 (see Sect. 2.2). (f) The corresponding ionospheric flux has been calculated mapping each flux value back to the ionosphere at $1000 \mathrm{~km}$, where the magnetic field is assumed to be $37 \mu \mathrm{T}$. (The total data set consists of 180000 points.)

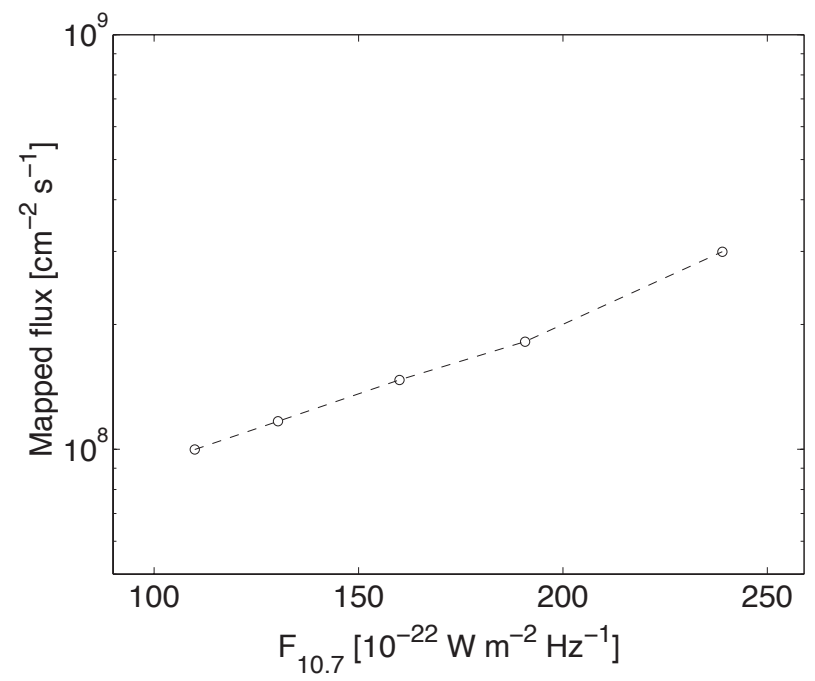

Fig. 9. Solar radiation dependence. The mapped ionospheric flux as a function of $\mathrm{F}_{10.7}$.

\subsection{Magnetic activity dependence}

The dependence of the mapped ionospheric flux on magnetic activity is evident for the frequently used indices $K_{\mathrm{p}}, \mathrm{AE}$ and $D_{s t}$. In Fig. 10, we display the outward flux plotted against 5 different values of $K_{\mathrm{p}}$ for low and high $\mathrm{F}_{10.7}$ values, respectively. Both groups show an increase, even though it is stronger for high $F_{10.7}$ values. Such a clear dependence on $K_{\mathrm{p}}$ has previously been observed on the Akebono and DE-1 spacecraft, as will be discussed in detail in Sect. 6.2.

\subsection{Solar wind parameter dependence}

In Fig. 11, we display the dependence on different solar wind parameters. Each parameter data set has been grouped into 5 different bins with equal number of data points. In each bin we have calculated the mean value of the parameter as well as that of the mapped ionospheric flux. The first column of Fig. 11 shows that the flux is fairly constant for the solar wind speed, but increases with increasing density, which is also evident when calculating the solar wind dynamic pressure, $n m v^{2}$. In the second column, the dependence on the 
interplanetary magnetic field (IMF) is shown. There is a strong dependence on the magnitude of the IMF. We also see that the outflow is higher for negative (southward) values of IMF $B_{Z, G S M}$, and when examining the parameter $-|v| B_{Z_{\mathrm{GSM}}}$, which approximates the dusk-ward (y) component of the solar wind electric field, we see an increase for positive values, which is expected. For higher values of $-\left[v \mid B_{Z_{\mathrm{GSM}}}\right.$, the reconnection rate at the magnetopause will be higher, which will lead to larger anti-sunward convection of the outflowing ions and thus larger flux. In addition, the geomagnetic activity is higher, which also yields an elevated flux.

The next column shows the dependence on the hourly standard deviation of the IMF, the solar wind density and temperature, respectively. We see an increase with the standard deviations of the IMF and the solar wind density, whereas with that of the solar wind temperature the increase is more moderate. In the last column we examine the dependence on the clock angle $\left(\theta_{c}=\arctan \left(B_{Y} / B_{Z}\right)\right)$, and two magnetosphereionosphere coupling functions. There is a strong dependence for large positive clock angles, which corresponds to large negative values of IMF $B_{Z}$. However, the dependence on negative IMF $B_{Z}$ should also translate to large negative values of the clock angle. The difference between negative and positive values of the clock angle should then be attributed to the sign of IMF $B_{Y}$, though we have no clear explanation why this should be the case. The coupling function $E_{\text {wav }}$ is defined as $v B_{\mathrm{T}} \sin ^{4}\left(\theta_{c} / 2\right)$, where $B_{\mathrm{T}}$ is the transverse component of the IMF. This gives a good measure of the crosspolar cap potential (Newell et al., 2006). Anti-sunward convection, and thus also centrifugal acceleration, of outflowing ions is directly related to the cross-polar cap potential, and therefore a strong dependence of the flux on $E_{\text {wav }}$ is expected. The correlation we find for low values of $E_{\mathrm{wav}}$, shown in Fig. 11k, is less evident compared to the results by Newell et al. (2006). The $\epsilon$ parameter (Perreault and Akasofu, 1978), $\epsilon=v B^{2} \sin ^{4}\left(\theta_{c} / 2\right)$, is another magnetosphereionosphere coupling function, which shows the same increase from the lowest to the highest parameter values as for $E_{\mathrm{wav}}$, but the increase is somewhat smoother.

\section{Discussion}

\subsection{Error discussion}

When quantifying the velocity and flux, variations should mainly be attributed to the statistical variance in the data, which can clearly be seen in Fig. 8. Possible error sources are: (1) the estimate of the outflow area and the magnetic field mapping ( $~ 50 \%$; the correction should mainly be towards larger areas as discussed in Sect. 2.1), (2) errors in the velocity calculation ( $<40 \%$, as described in Sect. 2.3 ), (3) the impossibility to distinguish between different ion species $(\sim 25 \%)$, and (4) uncertainties in the total density estimate.

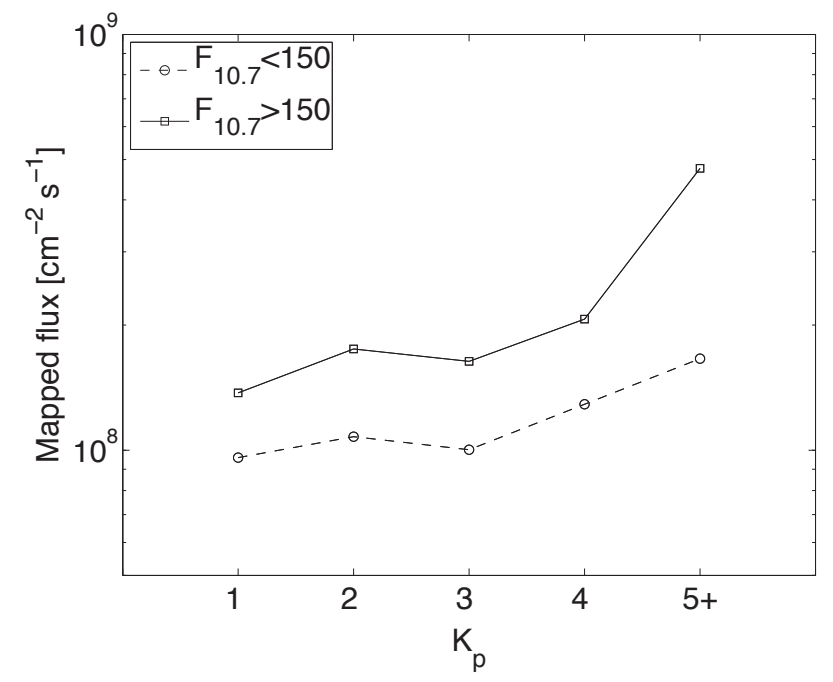

Fig. 10. Magnetic activity dependence. The mapped ionospheric flux as a function of $K_{\mathrm{p}}$.

The error in the original density estimate of Pedersen et al. (2008) is around $10-20 \%$, but this is mainly due to variations in the solar radiation. Our normalization to the daily variations of $\mathrm{F}_{10.7}$ should thus reduce this error. The possible errors (1)-(4) could add up to at most a factor of just above 2 , which is small compared to the statistical variance in the data.

The operation of EDI on Cluster 3 could possibly lead to an underestimation of the inferred density from the spacecraft potential. The high-energy electrons emitted from EDI will result in a higher spacecraft potential, and this is translated to a lower plasma density. In the investigated data set, the EDI current ranges from a few nA to $500 \mathrm{nA}$. Even for the highest currents the effect on the density estimate should be small. This can be understood, if we consider the current balance equation for the spacecraft:

$I_{\mathrm{ph}}+I_{\mathrm{EFW}}+I_{\mathrm{EDI}}=I_{\mathrm{e}}$,

where $I_{\mathrm{ph}}$ is the photoelectron current from the spacecraft, $I_{\mathrm{EFW}}$ is the bias current from the EFW probes and $I_{\mathrm{EDI}}$ is the EDI beam current. $I_{\mathrm{e}}$ is the electron current, which for a spacecraft smaller than the Debye length equals (Pedersen, 1995)

$I_{\mathrm{e}}=4 \pi r_{\mathrm{sc}}^{2} e \sqrt{\frac{K T_{\mathrm{e}}}{2 \pi m}} n\left(1+\frac{e V_{\mathrm{sc}}}{K T_{\mathrm{e}}}\right) \equiv C n\left(1+\frac{e V_{\mathrm{sc}}}{K T_{\mathrm{e}}}\right)$,

if we approximate the spacecraft as a spherical body with radius $r_{\mathrm{sc}}$. The density can then be related to the spacecraft potential as

$n=\frac{I_{\mathrm{ph}}+I_{\mathrm{EFW}}}{C\left(1+\frac{e V_{\mathrm{sc}}}{K T_{\mathrm{e}}}\right)}+\frac{I_{\mathrm{EDI}}}{C\left(1+\frac{e V_{\mathrm{sc}}}{K T_{\mathrm{e}}}\right)}$ 


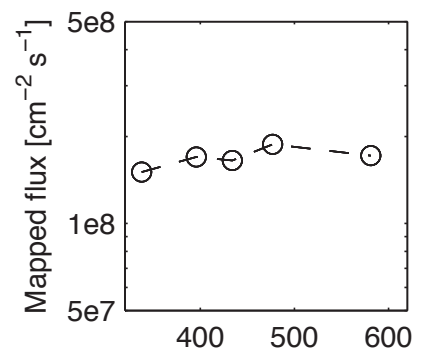

(a) $\mathrm{v}[\mathrm{km} / \mathrm{s}]$

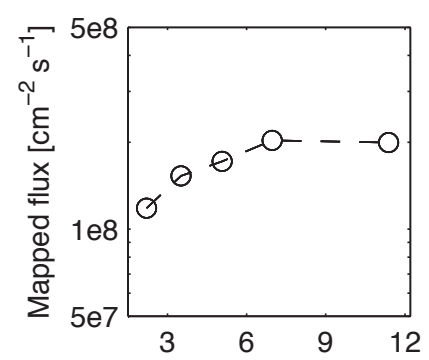

(b) $\mathrm{n}\left[\mathrm{cm}^{-3}\right]$

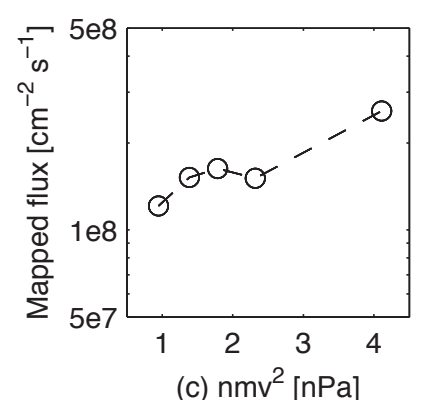

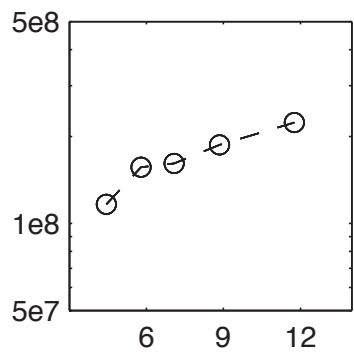

(d) IBI [nT]

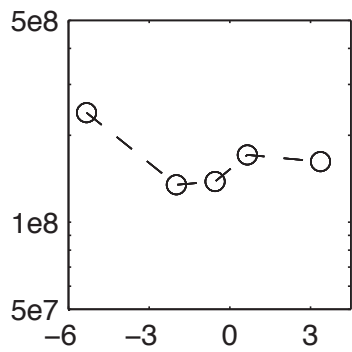

(e) $B_{Z, G S M}[n T]$

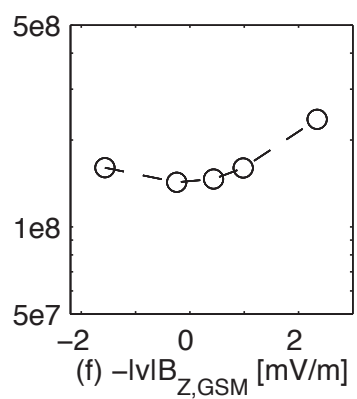

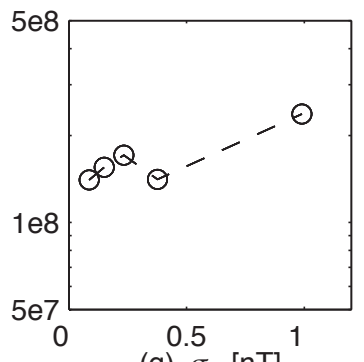

(g) $\sigma_{B}[n T]$

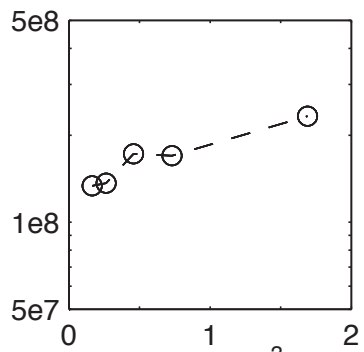

(h) $\sigma_{\mathrm{n}}\left[\mathrm{cm}^{-3}\right]$

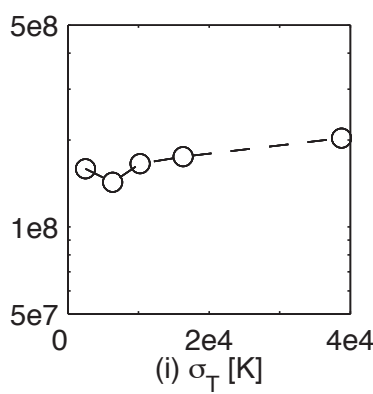

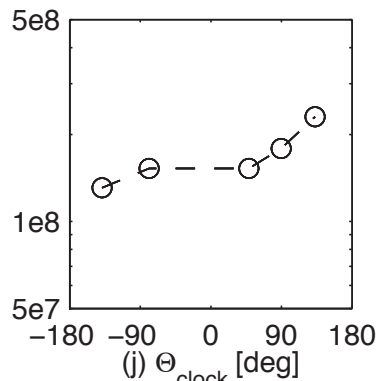
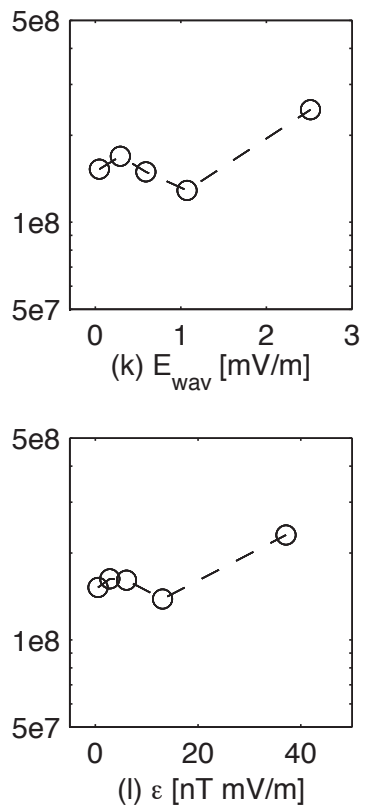

Fig. 11. Outflow dependence on different solar wind parameters. (a) Solar wind speed. (b) Solar wind density. (c) Solar wind dynamic pressure, $n m v^{2}$. (d) Magnitude of interplanetary magnetic field (IMF). (e) IMF $B_{Z, G S M}$. (f) Approximation of the y-component of the solar wind electric field, $E_{y} \approx-|v| B_{Z_{\mathrm{GSM}}}$. (g-i) Standard deviation of the IMF, the solar wind density and temperature. (j) The IMF clock angle. (k) The parameter $\mathrm{E}_{\mathrm{wav}}$, which is a measure of the cross-polar cap potential. (l) The $\epsilon$ parameter of Perreault and Akasofu (1978). (The total flux data set consists of 180000 points.)

The first term in Eq. (9) is modelled by the density relations in Eqs. (5) and (6), while the second term is responsible for the error in the density estimate due to EDI operation. In the lobes typical values of $V_{\mathrm{sc}}$ and $K T_{\mathrm{e}}$ are $20-40 \mathrm{~V}$ and a few $\mathrm{eV}$, respectively. For such conditions, a current on the order of $100 \mathrm{nA}$ will give rise to an underestimation of the density on the order of $0.01 \mathrm{~cm}^{-3}$. When examining the data, we can also confirm that the density distributions at different EDI currents only show small differences. We therefore consider errors related to the EDI current negligible; in any case they could only lead to a slight underestimate of density and flux.

As pointed out in Sect. 2.2, the $\mathrm{H}^{+} / \mathrm{O}^{+}$ratio in the ionospheric outflow (error (3)) depends on geomagnetic and solar activity with higher oxygen content during active times. The high-altitude study of the polar wind by Su et al. (1998), who observed a proton content of around $80 \%$, was based on solar minimum data with average $\mathrm{F}_{10.7}$ values well below 100. The geomagnetic activity during April-May 1996, when the measurements were conducted, was also low $\left(K_{\mathrm{p}}\right.$ around 2). In our data set, we have been forced to remove data with $\mathrm{F}_{10.7}$ below 100 (see Sect. 2.3). However, our mean values of $\mathrm{F}_{10.7}$ and $K_{\mathrm{p}}$ for the remaining data set are around $160 \times 10^{-22} \mathrm{~W} \mathrm{~m}^{-2} \mathrm{~Hz}^{-1}$ and 2.6, which shows that the overall activity still is moderate, justifying a low average oxygen content in the ionospheric outflows. In addition, for high oxygen content in the ionospheric outflow the wake signature will be small, since the ram kinetic energy of the oxygen ions is 16 times higher than for protons at the same velocity. The energy-per-charge of the $\mathrm{O}^{+}$ions will therefore normally be close to or even above the typical spacecraft potential. This results in a less enhanced wake and a smaller wake potential. As a consequence, our limit on the 
wake electric field of $2 \mathrm{mV} / \mathrm{m}$ will favour detection of cold ion flows with higher proton content than the real composition of the outflows. With these arguments in mind and since we have no good model of the $\mathrm{H}^{+} / \mathrm{O}^{+}$ratio as a function of $\mathrm{F}_{10.7}$ and $K_{\mathrm{p}}$, the ratio from the Su et al. (1998) study, which overlaps in altitude with our measurements, is a reasonable choice. It should also be noted that $\mathrm{Su}$ et al. (1998) most probably underestimate the proton content due to the potential screening effects as described in the next section.

Due to the dependence of the $\mathrm{H}^{+} / \mathrm{O}^{+}$ratio on geomagnetic and solar activity, a qualifier is needed for the results in Figs. 9 and 10, where the outflow increases clearly with $\mathrm{F}_{10.7}$ and $K_{\mathrm{p}}$. Part of this increase could result from overestimating the proton content during high geomagnetic and solar activity.

An alternative way to interpret our flux values, which circumvents the uncertainty in ion composition is to assume that the velocities of the ion species are similar, which is the case for centrifugal acceleration. In that case our flux values (divided by 0.8 ) can be seen as a lower limit to the total ion number flux. It is a lower limit since our method is less sensitive to flows with high oxygen content.

The error analysis performed on the velocity calculation and the limit of $40 \%$ reduce the number of data points to a third. To investigate whether this exclusion of points could introduce any systematic errors, we have reproduced all results using the total data set before error removal. The overall results are the same, showing that no systematic errors have been introduced.

As described in Sect. 2.2, the method relies on the existence of EDI data, which requires strong enough magnetic fields (above $30 \mathrm{nT}$ ). This will introduce a bias towards observations during stronger magnetic fields, and since the magnetic field decreases tailward more points are excluded the farther away the observations are made. The bias towards stronger magnetic fields in the tail could in turn favour observations during high negative values of IMF $B_{Z}$. Since the flux is higher for negative values of IMF $B_{Z}$, the bias could result in a slight overestimation of the median and mean values of the flux.

\subsection{Comparison to previously published results}

As was mentioned in the introductory section, there have been a large number of studies on ion outflow from the polar caps at low altitudes using different spacecraft: DE-1 (Nagai et al., 1984; Chandler et al., 1991), Akebono (Abe et al., 1993, 1996, 2004; Cully et al., 2003a), and Polar (Moore et al., 1997; Su et al., 1998; Chappell et al., 2000; Lennartsson et al., 2004; Liemohn et al., 2005; Huddleston et al., 2005; Peterson et al., 2006, 2008). The study at highest altitude so far was conducted by Su et al. (1998) using Polar data at apogee altitude at $8 R_{E}$ above the polar caps. Since we somewhat overlap with this study geographically, this is a good starting point for a comparison.
The Polar survey reports mean $\mathrm{H}^{+}$density of $0.3 \mathrm{~cm}^{-3}$ and velocity of $45 \mathrm{~km} / \mathrm{s}$. Our results are in general agreement with these values, even though our values are somewhat lower $\left(\left\langle n_{\mathrm{H}^{+}}\right\rangle=0.18 \mathrm{~cm}^{-3},\left\langle v_{\mathrm{H}^{+}}\right\rangle=26 \mathrm{~km} / \mathrm{s}\right)$. As pointed out by Engwall et al. (2009), the speed in our study should be higher than in the Polar study, since we measure farther down the tail and the speed should increase due to centrifugal acceleration and flux tube expansion. The explanation by Engwall et al. (2009) was that Su et al. (1998) probably miss a large part of the low-energy ions due to spacecraft potential shielding effects. Polar is equipped with a plasma source instrument, which reduces the spacecraft potential to around $+2 \mathrm{~V}$ to alleviate the shielding effects. However, $2 \mathrm{eV}$ corresponds to a flow speed of $20 \mathrm{~km} / \mathrm{s}$ for $\mathrm{H}^{+}$and protons with this flow velocity or below will not reach the spacecraft. As can be seen in Fig. 8b, the majority of the flows we measure have such low velocities.

When comparing the proton densities from the two studies, we see that our mean density (corrected by a factor of $0.8)$ at $8 R_{E}$ in Fig. $6 \mathrm{~b}$ is around the mean value of Polar of $0.3 \mathrm{~cm}^{-3}$, which could at first seem to be in contradiction with our previous conclusion that Polar would miss the ions with lowest energy. However, the Polar measurement was made essentially right above the poles, where the density should be higher, whereas our measurements at $8 R_{E}$ in general was made tailward from the Earth.

For comparison to other published values we use the global outflow of cold ions. Peterson et al. $(2006,2008)$ has put much effort in comparing measurements from spacecraft at different altitudes. The tables included in Peterson et al. (2008) and Engwall et al. (2009) show that the cold ion flow is higher than the ion flow with higher energy. Our mean outflow of $0.74 \times 10^{26}$ confirms this conclusion. The value is also very close to the cold ion outflow measured previously at low altitudes (Huddleston et al., 2005; Cully et al., 2003a; Nagai et al., 1984) (see Table 1 of Engwall et al. (2009)) and the total outflow at high altitude measured as flux by $\mathrm{Su}$ et al. (1998) and equated as total outflow by Peterson et al. (2008). This has several implications: (1) The cold ion outflows from the high-latitude ionosphere extend far back in the geomagnetic tail (to at least $19 R_{E}$ ). (2) This provides extensive evidence for the ionosphere as a major supplier of magnetospheric plasmas, as was first proposed by Chappell et al. (1987), and then supported in a number of global simulations (Chappell et al., 2000; Cully et al., 2003b; Huddleston et al., 2005). (3) The good agreement of the rate of ion outflow measured by two completely different methods also cross-validates both methods.

Huddleston et al. (2005) performed numerical simulations with initial conditions from Polar data to estimate the tail lobe density. The proton density was calculated to be $0.010-$ $0.16 \mathrm{~cm}^{-3}$ during quiet times, and $0.050-0.78 \mathrm{~cm}^{-3}$ during active times. Since measurements of cold ions with ion detectors are problematic, they provided no comparison to in situ measurements other than from ISEE (Eastman et al., 


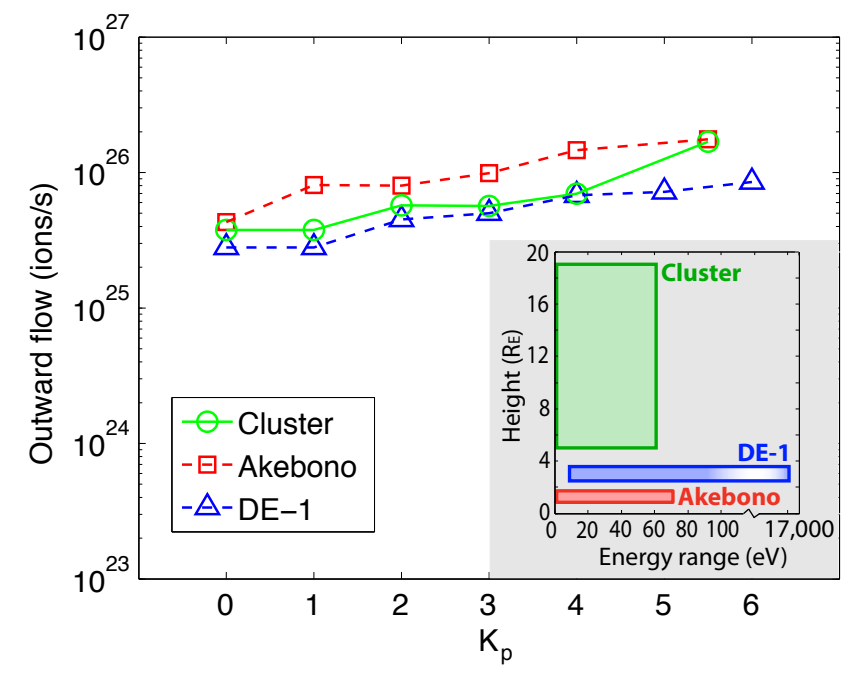

Fig. 12. Comparison to Akebono and DE-1. Comparison of the total ion outflow (ions/s) to measurements from DE-1 and Akebono for high $\mathrm{F}_{10.7}\left(\mathrm{~F}_{10.7}>150\right)$. The instrument energy ranges and altitudes covered by the spacecraft are displayed in the lower right corner. (Note that the spacecraft potential screening effect is not taken into account for Akebono and DE-1.)

1985), which detected densities between $10^{-3} \mathrm{~cm}^{-3}$ and $10^{-1} \mathrm{~cm}^{-3}$. The conclusion of Huddleston et al. (2005) was that a large fraction of the cold tail lobe ions are obscured to measurements by the spacecraft potential and that there exists a significant population of cold ions with energies of tens of $\mathrm{eV}$ or less in the tail lobes. Our measured mean density of $0.18 \mathrm{~cm}^{-3}$ is consistent with the numerically computed values and supports this conclusion.

DE-1 observed an altitude dependence of the velocity at low altitudes, where the velocity increased roughly from 3 to $11 \mathrm{~km} / \mathrm{s}$ from 1000 to $4000 \mathrm{~km}$ (Chandler et al., 1991). Abe et al. (1993) reported similar results from Akebono with velocities ranging roughly from 1 to $10 \mathrm{~km} / \mathrm{s}$ from 2000 to $10000 \mathrm{~km}$. In a more recent study, Abe et al. (2004) used Akebono data from almost ten years and got very similar results for high solar radiation, whereas the increase is lower for low solar radiation. In Fig. 6a, we see a clear increase of the velocity, even though it is not as steep as at lower altitudes. This is consistent with the faster expansion of the flux tube and larger curvature of the magnetic field lines at low altitudes than farther away from the Earth.

The dependencies on solar wind parameters and geomagnetic indices should be compared to similar studies on other spacecraft. Cully et al. (2003a) made an ambitious study of Akebono data sorting it to different solar wind conditions and geomagnetic indices. The strongest dependencies are found in the solar wind kinetic pressure, solar wind electric field, the IMF variability and the IMF magnitude. Strong correlation is also found to $K_{\mathrm{p}}$. This is qualitatively in accor- dance with our findings in Figs. 10 and 11. Lennartsson et al. (2004) presented a study on solar wind control of ion outflow, where significant enhancement of the ion outflow during southward IMF (increase by a factor of 2 for $\mathrm{H}^{+}$) were observed. There was also a strong dependence on the solar wind kinetic flow density and the Poynting flux. Those are related to the solar wind kinetic pressure and the parameter $E_{\text {wav }}$, respectively, for which we found strong outward flux correlation.

The flux dependence on $\mathrm{F}_{10.7}$ has the same shape as measured with Akebono (Cully et al., 2003a) for high values of $F_{10.7}$. The slight increase of the outward flux for low values of $F_{10.7}$ is not observed, but we are not able to accurately measure for $F_{10.7}$ values below 100. Abe et al. (2004) instead examined the ion outflow velocity dependence on $\mathrm{F}_{10.7}$ in Akebono data, which in contrast to the flux did not show any clear trend. This indicates that the solar radiation controls mainly the ion density, with higher ionization rates, and thus higher plasma densities, during periods of elevated solar radiation flux. Examining our data set, this hypothesis holds, since there is just a small difference ( 25 to $29 \mathrm{~km} / \mathrm{s}$ ) from the lowest to the highest $F_{10.7}$ values, whereas the density increases from 0.11 to $0.30 \mathrm{~cm}^{-3}$.

We now follow the example in Cully et al. (2003a) and make a comparison of the dependence of the total ion outflow on $K_{\mathrm{p}}$ for different spacecraft; DE-1 (Yau et al., 1988), Akebono (Cully et al., 2003a), and Cluster (current study). We restrict the comparison to high $\mathrm{F}_{10.7}\left(\mathrm{~F}_{10.7}>150\right)$, since this is the only region where our data overlap with the previously published results. The displayed values from Cluster are calculated from the mapped ionospheric flux times the constant polar cap area of $4.1 \times 10^{17} \mathrm{~cm}^{2}$. In reality, the polar cap area increases with increasing $K_{\mathrm{p}}$, which means that the increase in ions/s should have a stronger dependence on $K_{\mathrm{p}}$. Cully et al. (2003a) have adopted a variable polar cap area, while Yau et al. (1988) also integrate over a constant area. However, it should be noted that this area is bounded by invariant latitudes above $56^{\circ}$, thus much larger than the area used in this study $\left(\geq 70^{\circ}\right)$. In Fig. 12 , it can clearly be seen that all three spacecraft measurements give evidence for a strong $K_{\mathrm{p}}$ dependence. We see that they show the same qualitative, as well as quantitative, dependence on $K_{\mathrm{p}}$. Akebono is equipped with a suprathermal ion instrument detecting even the ions with lowest energies, whereas DE-1 misses the ions with lowest energies, due to the low-energy cut-off of the ion instrument $(10 \mathrm{eV})$ and the spacecraft potential screening effect, but instead includes ions with much larger energies (up to $17 \mathrm{keV}$ ).

\section{Conclusions}

The current study confirms the conclusions in Engwall et al. (2009) with an extended data set collected during 5 years in 
the declining phase of the solar cycle. They can be summarised as follows:

1. A cold ion population is detected throughout the lobes. As was shown by Engwall et al. (2009), this cold proton flux dominates over the outflow of more energetic $(>100 \mathrm{eV})$ protons.

2. The dominance in density is even stronger, since the low-energy ions have lower velocity. Our extensive data set has shown that there is a significant population of low-energy ions continuously present in the lobes, previously only predicted in simulations. The mean density from 5 to $20 R_{E}$ of the cold ions is $0.18 \mathrm{~cm}^{-3}$, which is much higher than expected from previous spacecraft missions, which have missed the large bulk of the cold ions.

3. There are almost no return fluxes in our measurements at high altitudes, which shows that most of the ions are escaping and not returning (Engwall et al., 2009). This was not known before, since the outflow previously only has been measured close to the Earth. Therefore, we now have a better estimate of the total loss than ever. The total outflow from the polar ionosphere is inferred to be of the order of $10^{26}$ ions/s.

4. The low energy of the ion outflow shows that it must originate in the ionosphere, and thus that the highlatitude ionospheric outflow continues far back in the tail. Our study also gives evidence for the ionosphere as a major supplier of magnetospheric plasma.

5. The low velocity and temperature of the ions even at high altitudes puts a strong limit on any acceleration or heating.

In addition, we have examined distribution in space of the outflow, as well as dependence on solar and magnetic activity, leading to the following conclusions:

6. The high-latitude low-energy outflow is concentrated in the central lobes, as can clearly be seen in Fig. 3. The rate of outflow is very low on the flanks. Along $Z_{\mathrm{GSM}}$ the occurrence of cold ions decreases towards the neutral sheet, where they get heated.

7. The outflow velocity increases approximately linearly with geocentric distance, while the density and flux decreases more like an exponential. This is consistent with centrifugal acceleration and increasing flux tube area.

8. The outflowing ions are convected in the dawn-to-dusk direction, due to the potential pattern in the polar cap.

9. The rate of ion outflow is dependent on $\mathrm{F}_{10.7}$, as well as on $K_{\mathrm{p}}$. The $K_{\mathrm{p}}$-dependence shows very good agreement with previous measurements at lower altitudes.
10. As for the solar wind influence, the driving parameters seem to be the solar wind dynamic pressure and the interplanetary magnetic field strength.

Acknowledgements. Magnetic field data from the Cluster FGM instrument (PI E. Lucek) have been used in the analysis. OmniWeb (http://omniweb.gsfc.nasa.gov/), the ACE and Wind teams, as well as NOAA's National Geophysical Data Center are acknowledged for making solar wind, solar radiation and magnetic activity data accessible. We also thank S. Haaland for valuable comments on the manuscript.

Topical Editor I. A. Daglis thanks A. Yau and another anonymous referee for their help in evaluating this paper.

\section{References}

Abe, T., Whalen, B. A., Yau, A. W., Horita, R. E., Watanabe, S., and Sagawa, E.: EXOS-D (Akebono) SMS Observations of the Polar Wind, J. Geophys. Res., 98, 11191-11203, 1993.

Abe, T., Watanabe, S., Whalen, B. A., Yau, A. W., and Sagawa, E.: Observations of Polar Wind and Thermal Ion Outflow by Akebono/SMS, J. Geomag. Geoel., 48, 319-325, 1996.

Abe, T., Yau, A. W., Watanabe, S., Yamada, M., and Sagawa, E.: Long-term variation of the polar wind velocity and its implication for the ion acceleration process: Akebono/Suprathermal ion mass spectrometer observations, J. Geophys. Res., 109, 93059317, 2004.

Axford, W. I.: The polar wind and the terrestrial helium budget, J. Geophys. Res., 73, 6855-6859, 1968.

Banks, P. M. and Holzer, T. E.: The polar wind, J. Geophys. Res., 73, 6846-6854, 1968.

Chandler, M. O., Moore, T. E., and Waite Jr., J. H.: Observations of polar ion outflows, J. Geophys. Res., 96, 1421-1428, 1991.

Chappell, C. R., Moore, T. E., and Waite, J. H.: The ionosphere as a fully adequate source of plasma for the Earth's magnetosphere, J. Geophys. Res., 92, 5896-5910, 1987.

Chappell, C. R., Giles, B. L., Moore, T. E., Delcourt, D. C., Craven, P. D., and Chandler, M. O.: The adequacy of the ionospheric source in supplying magnetospheric plasmas, J. Atmos. SolarTerr. Phys., 62, 421-436, 2000.

Cladis, J. B.: Parallel acceleration and transport of ions from polar ionosphere to plasma sheet, Geophys. Res. Lett., 13, 893-896, 1986.

Cully, C. M., Donovan, E. F., Yau, A. W., and Arkos, G. G.: Akebono/Suprathermal Mass Spectrometer observations of low-energy ion outflow: Dependence on magnetic activity and solar wind conditions, J. Geophys. Res., 108, 1093, doi:10.1029/2001JA009200, 2003a.

Cully, C. M., Donovan, E. F., Yau, A. W., and Opgenoorth, H. J.: Supply of thermal ionospheric ions to the central plasma sheet, J. Geophys. Res., 108, 1092, doi:10.1029/2002JA009457, 2003b.

Delcourt, D. C., Chappell, C. R., Moore, T. E., and Waite Jr., J. H.: A three-dimensional numerical model of ionospheric plasma in the magnetosphere, J. Geophys. Res., 94, 11893-11920, 1989.

Eastman, T. E., Frank, L. A., and Huang, C. Y.: The boundary layers as the primary transport regions of the Earth's magnetotail, J. Geophys. Res., 90, 9541-9560, 1985.

Engwall, E., Eriksson, A. I., André, M., Dandouras, I., Paschmann, G., Quinn, J., and Torkar, K.: Low-energy (order $10 \mathrm{eV}$ ) ion flow 
in the magnetotail lobes inferred from spacecraft wake observations, Geophys. Res. Lett., 33, 6110-6113, corrected in Geophys. Res. Lett., 33, 14102 (2006), 2006a.

Engwall, E., Eriksson, A. I., and Forest, J.: Wake formation behind positively charged spacecraft in flowing tenuous plasmas, Phys. Plasmas, 13, 2904-2913, 2006b.

Engwall, E., Eriksson, A. I., Cully, C. M., André, M., Torbert, R., and Vaith, H.: Earth's ionospheric outflow dominated by hidden cold plasma, Nature Geosci., 2, 24-27, 2009.

Eriksson, A. I. and Winkler, E.: Photoemission current and solar EUV radiation: Cluster and TIMED observations, in: Proceedings of the 10th Spacecraft Charging Technology Conference, 2008.

Eriksson, A. I., André, M., Klecker, B., Laakso, H., Lindqvist, P.-A., Mozer, F., Paschmann, G., Pedersen, A., Quinn, J., Torbert, R., Torkar, K., and Vaith, H.: Electric field measurements on Cluster: comparing the double-probe and electron drift techniques, Ann. Geophys., 24, 275-289, 2006, http://www.ann-geophys.net/24/275/2006/.

Gustafsson, G., Bostrom, R., Holback, B., Holmgren, G., Lundgren, A., Stasiewicz, K., Ahlen, L., Mozer, F. S., Pankow, D., Harvey, P., Berg, P., Ulrich, R., Pedersen, A., Schmidt, R., Butler, A., Fransen, A. W. C., Klinge, D., Thomsen, M., Fälthammar, C.G., Lindqvist, P.-A., Christenson, S., Holtet, J., Lybekk, B., Sten, T. A., Tanskanen, P., Lappalainen, K., and Wygant, J.: The Electric Field and Wave Experiment for the Cluster Mission, Space Sci. Rev., 79, 137-156, 1997.

Haaland, S. E., Paschmann, G., Förster, M., Quinn, J. M., Torbert, R. B., McIlwain, C. E., Vaith, H., Puhl-Quinn, P. A., and Kletzing, C. A.: High-latitude plasma convection from Cluster EDI measurements: method and IMF-dependence, Ann. Geophys., 25, 239-253, 2007, http://www.ann-geophys.net/25/239/2007/.

Hirahara, M., Mukai, T., Terasawa, T., Machida, S., Saito, Y., Yamamoto, T., and Kokubun, S.: Cold dense ion flows with multiple components observed in the distant tail lobe by Geotail, J. Geophys. Res., 101, 7769-7784, 1996.

Hoffman, J. H.: Studies of the composition of the ionosphere with a magnetic deflection mass spectrometer, Int. J. Mass Spectrom. Ion Phys., 4, 315-322, 1970.

Hoffman, J. H. and Dodson, W. H.: Light ion concentrations and fluxes in the polar regions during magnetically quiet times, J. Geophys. Res., 85, 626-632, 1980.

Hoffman, J. H., Dodson, W. H., Lippincott, C. R., and Hammack, H. D.: Initial ion composition results from the ISIS 2 satellite, J. Geophys. Res., 79, 4246-4251, 1974.

Huddleston, M. M., Chappell, C. R., Delcourt, D. C., Moore, T. E., Giles, B. L., and Chandler, M. O.: An examination of the process and magnitude of ionospheric plasma supply to the magnetosphere, J. Geophys. Res., 110, A12202, doi:10.1029/2004JA010401, 2005.

Khotyaintsev, Y., Lindqvist, P.-A., Eriksson, A., and André, M.: The EFW Data in the CAA, in: Cluster CAA book, in press, 2009.

Laakso, H., Pfaff, R., and Janhunen, P.: Polar observations of electron density distribution in the Earths magnetosphere. 1. Statistical results, Ann. Geophys., 20, 1711-1724, 2002,

http://www.ann-geophys.net/20/1711/2002/.

Lennartsson, O. W., Collin, H. L., and Peterson, W. K.: Solar wind control of Earth's $\mathrm{H}^{+}$and $\mathrm{O}^{+}$outflow rates in the 15$\mathrm{eV}$ to 33-keV energy range, J. Geophys. Res., 109, A12212, doi:10.1029/2005RG000194, 2004.

Liemohn, M. W., Moore, T. E., Craven, P. D., Maddox, W., Nagy, A. F., and Kozyra, J. U.: Occurrence statistics of cold, streaming ions in the near-Earth magnetotail: Survey of Polar-TIDE observations, J. Geophys. Res., 110, 7211-7226, 2005.

Lockwood, M., Chandler, M. O., Horwitz, J. L., Waite Jr., J. H., Moore, T. E., and Chappell, C. R.: The cleft ion fountain, J. Geophys. Res., 90, 9736-9748, 1985.

Moore, T. E. and Horwitz, J. L.: Stellar ablation of planetary atmospheres, Rev. Geophys., 45, RG3002, doi:10.1029/2004JA010563, 2007.

Moore, T. E., Chappell, C. R., Chandler, M. O., Craven, P. D., Giles, B. L., Pollock, C. J., Burch, J. L., Young, D. T., Waite Jr., J. H., Nordholt, J. E., Thomsen, M. F., McComas, D. J., Berthelier, J. J., Williamson, W. S., Robson, R., and Mozer, F. S.: Highaltitude observations of the polar wind, Science, 277, 349-351, 1997.

Moore, T. E., Chandler, M. O., Chappell, C. R., Comfort, R. H., Craven, P. D., Delcourt, D. C., Elliot, H. A., Giles, B. L., Horwitz, J. L., Pollock, C. J., and Su, Y.-J.: Polar/TIDE Results on Polar Ion Outflows, in: Sun-Earth Plasma Connections, Geophysical Monograph 109, pp. 87-101, American Geophysical Union, 1999a.

Moore, T. E., Fok, M.-C., Chandler, M. O., Chappell, C. R., Christon, S. P., Delcourt, D. C., Fedder, J., Huddleston, M., Liemohn, M., Peterson, W. K., and Slinker, S.: Plasma sheet and (nonstorm) ring current formation from solar and polar wind sources, J. Geophys. Res., 110, A02210, doi:10.1029/2006JA011731, 2005.

Mukai, T., Hirahara, M., Machida, S., Saito, Y., Terasawa, T., and Nishida, A.: Geotail observation of cold ion streams in the medium distance magnetotail lobe in the course of a substorm, Geophys. Res. Lett., 21, 1023-1026, 1994.

Nagai, T., Waite, J. H., Green, J. L., Chappell, C. R., Olsen, R. C., and Comfort, R. H.: First measurements of supersonic polar wind in the polar magnetosphere, Geophys. Res. Lett., 11, 669672, 1984.

Newell, P. T., Sotirelis, T., Liou, K., Meng, C.-I., and Rich, F. J.: Cusp latitude and the optimal solar wind coupling function, J. Geophys. Res., 111, A09207, doi:10.1029/2007JA012636, 2006.

Olsen, R. C.: The hidden ion population of the magnetosphere, J. Geophys. Res., 87, 3481-3488, 1982.

Paschmann, G., Melzner, F., Frenzel, R., Vaith, H., Parigger, P., Pagel, U., Bauer, O. H., Haerendel, G., Baumjohann, W., Scopke, N., Torbert, R. B., Briggs, B., Chan, J., Lynch, K., Morey, K., Quinn, J. M., Simpson, D., Young, C., McIlwain, C. E., Fillius, W., Kerr, S. S., Mahieu, R., and Whipple, E. C.: The Electron Drift Instrument for Cluster, Space Sci. Rev., 79, 233-269, 1997.

Pedersen, A.: Solar wind and magnetosphere plasma diagnostics by spacecraft electrostatic potential measurements, Ann. Geophys., 13, 118-129, 1995, http://www.ann-geophys.net/13/118/1995/.

Pedersen, A., Mozer, F., and Gustafsson, G.: Electric Field Measurements in a Tenuous Plasma with Spherical Double Probes, in: Measurement Techniques in Space Plasmas: Fields, Geophysical Monograph 103, pp. 1-12, American Geophysical 
Union, 1998.

Pedersen, A., Lybekk, B., André, M., Eriksson, A. I., Masson, A., Mozer, F. S., Lindqvist, P., Decreau, P., Dandouras, I., Sauvaud, J.-A., Fazakerley, A. N., Taylor, M., Paschmann, G., Svenes, K., Torkar, K. M., , and Whipple, E. C.: Electron density estimations derived from spacecraft potential measurements on Cluster in tenuous plasma regions, J. Geophys. Res., 113, A07S33, doi:10.1029/2008JA013059, 2008.

Perreault, P. and Akasofu, S.-I.: A study of geomagnetic storms., Geophys. J. R. Astron. Soc., 54, 547-573, 1978.

Peterson, W. K., Collin, H. L., Lennartsson, O. W., and Yau, A. W.: Quiet time solar illumination effects on the fluxes and characteristic energies of ionospheric outflow, J. Geophys. Res., 111, 11-25, 2006.

Peterson, W. K., Andersson, L., Callahan, B. C., Collin, H. L., Scudder, J. D., and Yau, A. W.: Solar-minimum quiet-time ion energization and outflow in dynamic boundary related coordinates, J. Geophys. Res., 113, A07222, doi:10.1029/2007JA012636, 2008.

Rème, H., Aoustin, C., Bosqued, J. M., et al.: First multispacecraft ion measurements in and near the Earth's magnetosphere with the identical Cluster ion spectrometry (CIS) experiment, Ann. Geophys., 19, 1303-1354, 2001, http://www.ann-geophys.net/19/1303/2001/.

Seki, K., Terasawa, T., Hirahara, M., and Mukai, T.: Quantification of tailward cold $\mathrm{O}^{+}$beams in the lobe/mantle regions with Geotail data: Constraints on polar $\mathrm{O}^{+}$outflows, J. Geophys. Res., 103, 29371-29382, 1998.

Seki, K., Hirahara, M., Hoshino, M., Terasawa, T., Elphic, R. C., Saito, Y., Mukai, T., Hayakawa, H., Kojima, H., and Matsumoto, H.: Cold ions in the hot plasma sheet of Earth's magnetotail, Nature, 422, 589-591, 2003.
Su, Y.-J., Horwitz, J. L., Moore, T. E., Giles, B. L., Chandler, M. O., Craven, P. D., Hirahara, M., and Pollock, C. J.: Polar wind survey with the Thermal Ion Dynamics Experiment/Plasma Source Instrument suite aboard POLAR, J. Geophys. Res., 103, 29305 29337, 1998.

Svenes, K. R., Lybekk, B., Pedersen, A., and Haaland, S.: Cluster observations of near-Earth magnetospheric lobe plasma densities - a statistical study, Ann. Geophys., 26, 2845-2852, 2008, http://www.ann-geophys.net/26/2845/2008/.

Tam, S. W. Y., Yasseen, F., Chang, T., and Ganguli, S. B.: Selfconsistent kinetic photoelectron effects on the polar wind, Geophys. Res. Lett., 22, 2107-2110, 1995.

Torkar, K., Riedler, W., Escoubet, C. P., Fehringer, M., Schmidt, R., Grard, R. J. L., Arends, H., Rdenauer, F., Steiger, W., Narheim, B. T., Svenes, K., Torbert, R., André, M., Fazakerley, A., Goldstein, R., Olsen, R. C., Pedersen, A., Whipple, E., and Zhao, H.: Active spacecraft potential control for Cluster - implementation and first results, Ann. Geophys., 19, 1289-1302, 2001, http://www.ann-geophys.net/19/1289/2001/.

Tsyganenko, N. A.: Modeling the Earth's magnetospheric magnetic field confined within a realistic magnetopause, J. Geophys. Res., 100, 5599-5612, 1995.

Yau, A. W. and André, M.: Sources of ion outflow in the high latitude ionosphere, Space Sci. Rev., 80, 1-25, 1997.

Yau, A. W., Peterson, W. K., and Shelley, E. G.: Quantitative Parametrization of Energetic Ionospheric Ion Outflow, in: Modeling Magnetospheric Plasma, Geophysical Monograph 44, pp. 211-217, American Geophysical Union, 1988.

Yau, A. W., Abe, T., and Peterson, W. K.: The polar wind: Recent observations, J. Atmos. Sol.-Ter. Phys., 69, 1936-1983, 2007. 